\title{
1 Functional ultrasound speckle decorrelation-based velocimetry of the brain
}

2 Jianbo Tang ${ }^{1}$, Dmitry D. Postnov ${ }^{1,3}$, Kivilcim Kilic ${ }^{1}$, Sefik Evren Erdener ${ }^{1}$, Blaire Lee ${ }^{1}$, John T.

3 Giblin ${ }^{1}$, Thomas L. Szabo ${ }^{1}$ \& David A. Boas ${ }^{1, *}$.

4 Dr. J. Tang, D. D. Postnov, K. Kilic, S. E. Erdener, Ms. B. Lee, Mr. J. T. Giblin, Prof. T. L. Szabo, 5 D. A. Boas

6 Neurophotonics Center, Department of Biomedical Engineering, Boston University, Boston, MA

$7 \quad 02215$, USA

8 E-mail: dboas@bu.edu

9 Dr. D. D. Postnov

10 Biomedical Sciences Institute, Copenhagen University, Copenhagen, 2200, Denmark

12 Keywords: functional ultrasound velocimetry, field autocorrelation function, brain imaging, 13 cerebral blood flow velocity

\section{Abstract:}

16 A high-speed, contrast free, quantitative ultrasound velocimetry (vUS) for blood flow velocity

17 imaging throughout the rodent brain is developed based on the normalized first order temporal

18 autocorrelation function of the ultrasound field signal. vUS is able to quantify blood flow velocity

19 in both transverse and axial directions, and is validated with numerical simulation, phantom

20 experiments, and in vivo measurements. The functional imaging ability of vUS is demonstrated by

21 monitoring blood flow velocity changes during whisker stimulation in awake mice. Compared to

22 existing power Doppler and color Doppler-based functional ultrasound imaging techniques, vUS

23 shows quantitative accuracy in estimating both axial and transverse flow speeds and resistance to

24 acoustic attenuation and high frequency noise. 


\section{Introduction}

Functional quantitative in vivo imaging of the entire brain with high spatial and temporal resolution remains an open quest in biomedical imaging. Current available methods are limited either by shallow penetration of optical microscopies that only allow imaging of superficial cortical layers, or by low spatiotemporal resolution such as functional magnetic resonance imaging or positron emission tomography. Ultrasound-based blood flow imaging techniques hold the promise to fulfill the unmet needs ${ }^{[1,2]}$, particularly with the emerging implementation of ultrafast ultrasound plane wave emission $^{[3]}$ which paves the way for ultrasound to be applied for functional cerebral hemodynamic imaging of the entire rodent brain with 10-100 $\mu \mathrm{m}$ resolution.

Since the introduction of ultrafast plane wave emission-based Power Doppler functional ultrasound imaging (PD-fUS $)^{[4]}$, an increasing number of studies are exploiting the capabilities of PD-fUS for functional brain imaging studies ${ }^{[5-7]}$. However, the exact relationship between the PD-fUS signal and the underlying physiological parameters is quite complex as the PD-fUS signal is also affected by the acoustic attenuation, beam pattern, clutter rejection and flow speed, in addition to the blood volume fraction and hematocrit ${ }^{[8,9]}$. On the other hand, ultrasound Color Doppler (CD-fUS) is able to measure a specific physiological parameter of the axial blood flow velocity but suffers from unstable estimations of mean speed due to the presence of noise and from incorrect estimation if opposite flows exist within the measurement voxel ${ }^{[2,4,10-12]}$. The microbubble tracking-based ultrasound localization microscopy $\left(\mathrm{ULM}^{[13]}\right)$ method is able to map the whole mouse brain vasculature (coronal plane) and quantify the in-plane blood flow velocity (vULM ${ }^{[13,14]}$ ) with $\sim 10$ $\mu m$ resolution. However, it suffers from a fundamental limitation of low temporal resolution as it requires extended data acquisition periods $\left(\sim 150\right.$ seconds for 75,000 images $\left.{ }^{[13]}\right)$ to accumulate 
47 sufficient microbubble events to form a single vascular image and corresponding velocity map,

48 limiting its potential for functional brain imaging studies.

49 Here, we report a novel ultrasound speckle decorrelation-based velocimetry (vUS) method for

50 blood flow velocity image of the rodent brain that overcomes the aforementioned limitations. We

51 derived vUS theory which shows that the ultrasound field signal decorrelation in small vessels is

52 not only determined by flow speed but also the axial velocity gradient and a phase term due to axial

53 movement. We further developed a comprehensive experimental implementation and data

54 processing methodology to apply vUS for blood flow velocity imaging of the rodent brain with

55 high spatiotemporal resolution and without the need for exogenous contrast. We validated vUS

56 with numerical simulations, phantom experiments, and in vivo measurements, and demonstrated

57 the functional imaging ability of vUS by quantifying blood flow velocity changes during whisker

58 stimulation in awake mice. We further show its advantage over PD-fUS and CD-fUS in terms of

59 quantitative accuracy in estimating axial and transverse flow speeds and its resistance to acoustic

60 attenuation and high frequency noise through phantom and in vivo measurements.

\section{Results}

\section{1. vUS theory}

The time varying ultrasound signal detected from a measurement voxel at time $t$ can be considered as the integration of all moving point scatters within the voxel, and the ultrasound pressure arising from a given voxel can thus be written as,

$$
\operatorname{sIQ}\left(x_{0}, y_{0}, z_{0}, t\right)=R \sum_{i_{S}}^{N_{S}} e^{-\frac{\left(x_{i_{S}}(t)-x_{0}\right)^{2}}{2 \sigma_{x}^{2}}-\frac{\left(y_{i_{S}}(t)-y_{0}\right)^{2}}{2 \sigma_{y}^{2}}-\frac{\left(z_{i_{S}}(t)-z_{0}\right)^{2}}{2 \sigma_{z}^{2}}} e^{i 2 k_{0}\left(z_{i_{S}}(t)-z_{0}\right)}
$$


71 intensity of the point spread function (PSF) in $x, y$, and $z$ directions, respectively; and $k_{0}$ is the
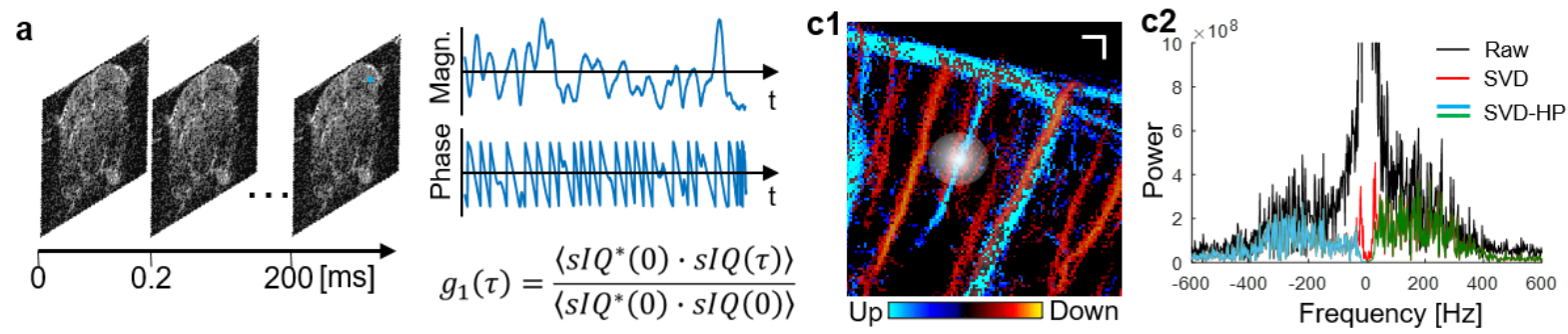

b1

b2

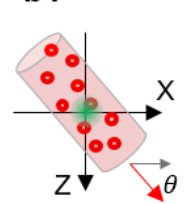

b3

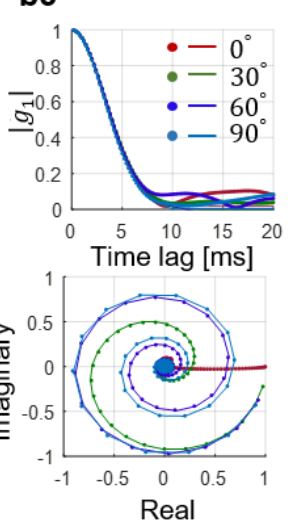

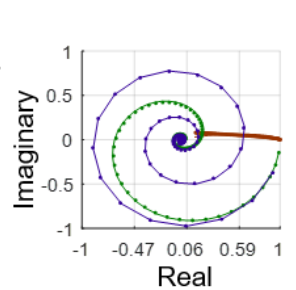

d
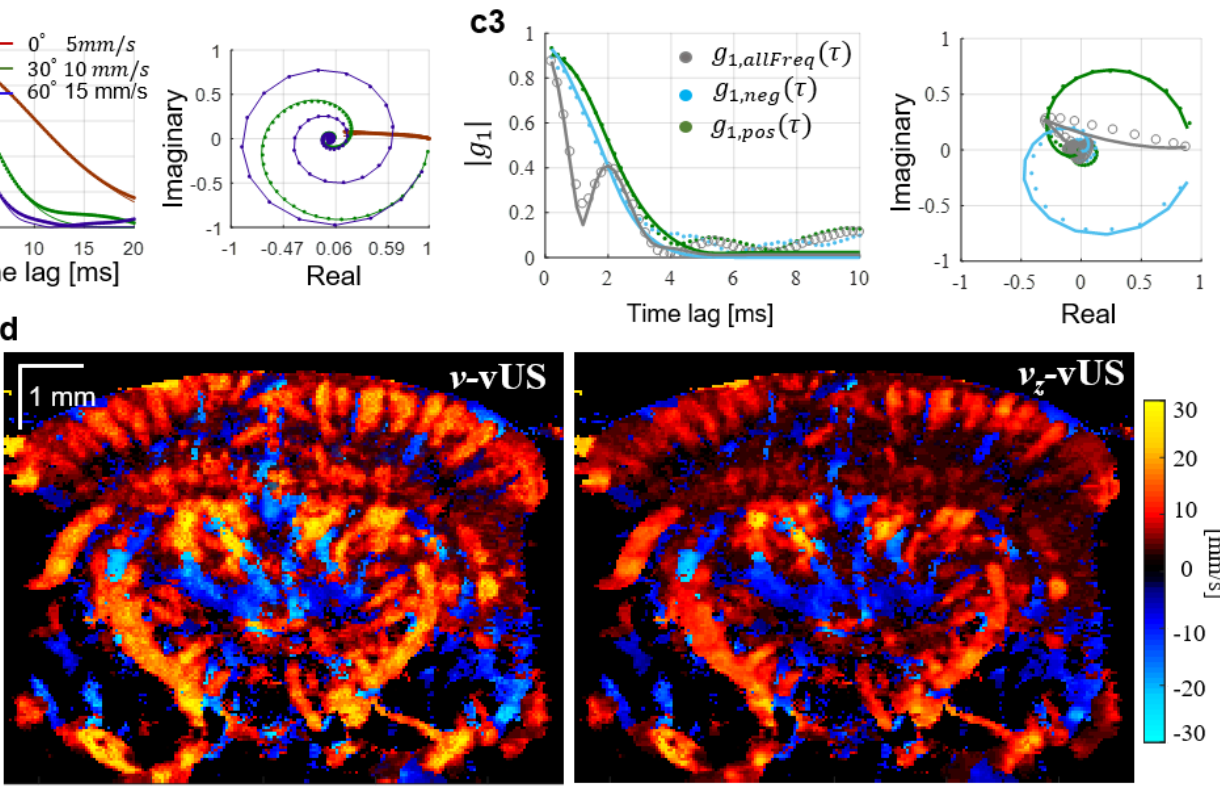

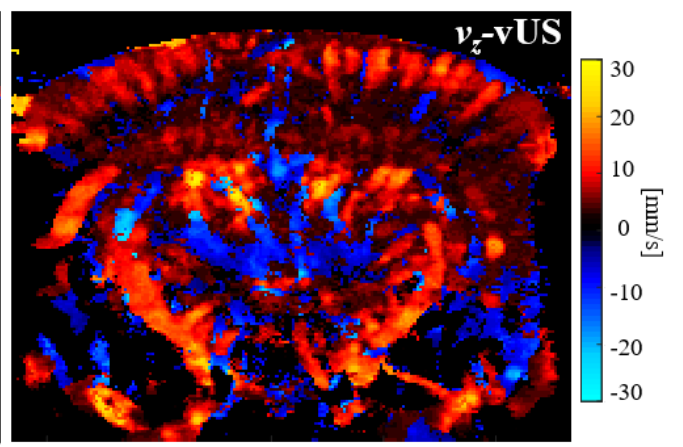

75 Figure 1 Principle of ultrasound field speckle decorrelation-based velocimetry (vUS). (a) A time series of

a high frame rate complex ultrasound quadrature signal after bulk motion rejection $(s I Q(t))$ was used for $g_{1}(\tau)$ calculation. (b) Characteristics of $g_{1}(\tau)$; (b1) Scatterers flow through the measurement voxel at an angle $\theta$; Magnitude decorrelation of $\left|g_{1}(\tau)\right|$ and field decorrelation of $g_{1}(\tau)$ in the complex plane at (b2) different angles with different speeds and (b3) different angles with the same speed $\left(v_{0}=15 \mathrm{~mm} / \mathrm{s}\right)$. (c1) 
81 ultrasound point spread function; (c2) Frequency power spectrum from in vivo data where descending and

82 ascending vessels were observed in the same measurement voxel; (c3) $g_{1}(\tau)$ calculated using whole

83 frequency signal (gray circles), negative frequency signal (cyan dots), and positive frequency signal (green

84 dots), respectively. (d) Representative total velocity map and axial velocity map reconstructed with vUS of

85 a mouse brain; descending flow map is overlapped on the ascending flow map. The solid lines in (b\&c) are

86 the fitted $g_{1}(\tau)$ using Equation 3.

As shown in Figure 1a, the movement of particles will cause the detected ultrasound field signal

to fluctuate in both magnitude and phase. This movement can be quantified based on the dynamic

of a time varying ultrasound signal for a measurement voxel is given by,

$$
g_{1}(\tau)=E\left[\frac{\left\langle\operatorname{sI} Q^{*}(t) \operatorname{sIQ}(t+\tau)\right\rangle_{t}}{\langle\operatorname{sIQ}(t) \operatorname{sIQ}(t)\rangle_{t}}\right]
$$

where, $\tau$ is the time lag; $\mathrm{E}[\ldots]$ indicates the average over random initial positions; $\langle\ldots\rangle_{t}$ represents

complex conjugate. Figure 1b illustrates the major characteristics of $g_{1}(\tau)$. Briefly, 1) $g_{1}(\tau)$

0) in the complex plane, and 3) different flow angle has different decorrelation path in the complex

plane, as shown in Figure 1b2. The rotating decorrelation in the complex plane is caused by the phase change due to axial movement. As shown in Figure 1b3, flows with the same total speed but in different angles have the same magnitude decorrelation (left panel) but different 'rotation paths' in the complex plane (right panel). This feature gives $g_{1}(\tau)$ analysis the ability to recover both axial velocity component and total flow speed.

When imaging the cerebral vasculature, the blood vessel diameter is usually less than the ultrasound system point spread function as indicated by Figure 1c1. In this case, the group velocity and velocity distribution must be taken into account as the relative movement of the scattering particles 
105

106

107

108

109

110

111

112

113

114

115

116

117

118

119

120

121

122

123

124

125

126

127

will result in additional decorrelation ${ }^{[15]}$. To simplify the derivation, we used a Gaussian speed distribution where, $v_{g p}$ is the group velocity; and $\sigma_{v}$ describes the velocity distribution, and we finally arrive at,

From Equation3, we note that in addition to flow speed, the axial velocity distribution $\sigma_{v z}$ also contributes to the magnitude decorrelation, and the axial velocity component leads to a phase term in $g_{1}(\tau)$ decorrelation. For details regarding the theoretical derivation, please refer to the

\section{Experimental Section-vUS theory derivation.}

In addition, we noticed from the in vivo data that it's common to have opposite flows present in the same measurement voxel when imaging the rodent brain, as shown in Figure 1c1. In this case, $g_{1}(\tau)$ is a mix of dynamics of opposite flows and behaves very differently from that of the single direction flow as can be observed from Figs. 1 b2 vs c3 (gray circles). In addition, we observed that the majority of the mouse cerebral blood vessels contain an axial velocity component to the flow. This axial flow component causes the frequency spectrum to shift to negative values if the flow is away from the transducer, and positive if the flow is towards the transducer. Thus, we used a directional filter (positive-negative frequency separation) method to obtain the positive frequency and negative frequency signals for the $g_{1}(\tau)$ calculation, as shown in Figure 1c2.

To implement the vUS technology, we developed a comprehensive vUS data acquisition and processing method (Materials and Methods-vUS implementation and Figure S1). Figure 1d shows representative in-plane total velocity and axial velocity maps of a mouse brain reconstructed by vUS. The descending flow velocity map which is reconstructed from the negative frequency component $\left(s I Q_{n e g}\right)$ is overlapped on the ascending flow velocity map which is obtained from the positive frequency component $\left(s I Q_{\text {pos }}\right)$. Like the existing PD-fUS and CD-fUS techniques, vUS 
has an in-plane spatial resolution of $\sim 100 \mu \mathrm{m}$ which is determined by the ultrasound system

acquisition parameters. Figure S2 shows more vUS results at different coronal planes.

\subsection{Validation of vUS}

131 The numerical simulation validation (details in Materials and Methods) results shown in Figure

2a suggest that the vUS reconstructed total velocity $(v)$, transverse velocity component $\left(v_{x}\right)$ and

133 axial velocity component $\left(v_{z}\right)$ agree well with preset speeds and angles. It is worth noting that vUS

134 is capable of measuring transverse flows (i.e. $\theta=0^{\circ}$ ) and differentiating the axial velocity

135 component from the transverse velocity component for the angled flows, as shown by results from

136 flow angle $\theta=30^{\circ}$ and $\theta=60^{\circ}$. For all simulation results, the correlation coefficient between

$v_{\text {set }}$ and $v_{\text {fit_mean }}$ were $\mathrm{r}>0.99$ with $\mathrm{p}<0.001$.

\section{a Numerical}

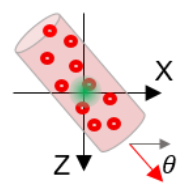

b1 Phantom
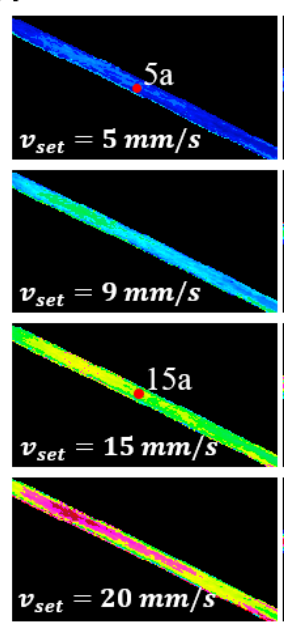
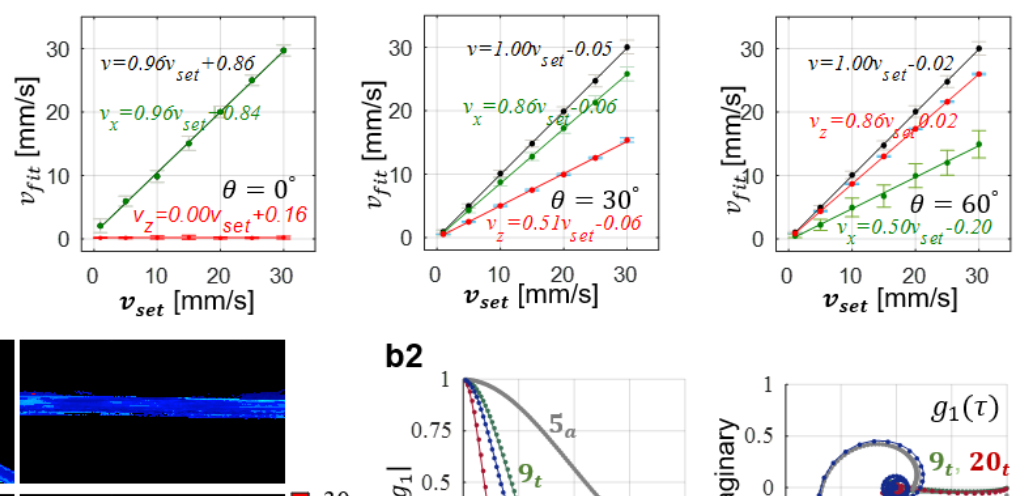

$9 \mathrm{t}$

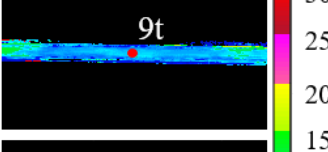

b2
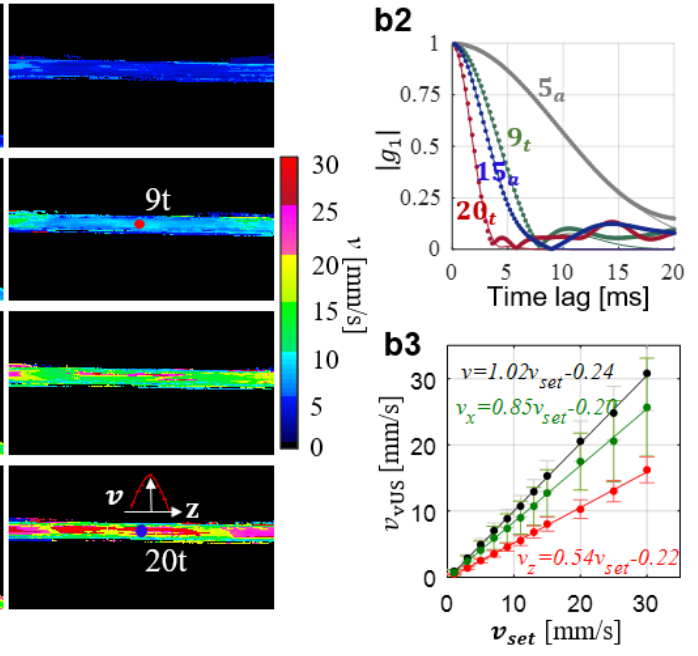

Figure 2 vUS numerical and phantom validation. (a) Numerical simulation validation with different flowing angles and speeds. Error bars: standard deviation. (b) Phantom validation of blood flowing through angled 
141 and transverse positioned micro tubes (inner diameter $580 \mu \mathrm{m}$ ). (b1) vUS reconstructed velocity maps of

142 angled and transverse flows at different speeds. The inset in the right bottom panel shows the cross sectional

143 laminar velocity profile of the transverse flow. (b2) Experimental $g_{1}(\tau)$ (dots) and corresponding vUS

144 fit results (solid lines) for both angled and transverse flows at different speeds. (b3) Results of vUS

$145\left(v, v_{x}\right.$ and $\left.v_{z}\right)$ for transverse flow $\left(\theta \approx 0^{\circ}\right.$, left $)$ and angled flow $\left(\theta \approx 30^{\circ}\right.$, right $)$. Error bars: standard

146 deviation.

147 The phantom validation experiments (details in Materials and Methods) were performed with

148 blood samples flowing through a micro plastic tube buried within a static agarose phantom, as

149 shown in Figure 2b. Figure $2 \mathbf{b} 1$ shows the velocity maps of both angled and transverse flows at

150 preset speeds of 5, 9, 15, and $20 \mathrm{~mm} / \mathrm{s}$. A laminar velocity profile was observed, particularly for

151 higher flow speeds, as indicated in the inset of Figure 2b1. Figure $2 \mathbf{b} 2$ shows the experimental

152 (dots) and vUS fitted $g_{1}(\tau)$, from which we see that $g_{1}(\tau)$ decays faster for higher speeds, and, as

153 shown in the complex plane, $g_{1}(\tau)$ rotates and decays to $(0,0)$ for angled flows $\left(5_{a}\right.$ and $\left.15_{a}\right)$ which

154 is due to the axial velocity component inducing a phase shift as indicated in Equation 3. Different

155 flow angles will have different 'rotation paths' in the complex plane. Figure $2 \mathbf{b} 3$ shows the vUS

156 reconstructed results compared to preset speeds, from which we note that the vUS measurements

157 of total speed agree well with the preset speeds even for speeds as low as $1 \mathrm{~mm} / \mathrm{s}$ for both transverse

158 and angled flows. The correlation coefficient between $v_{\text {set }}$ and $v_{\text {fit_mean }}$ for transverse and angled

159 flows were $r>0.99$ with $\mathrm{p}<0.001$. Figure $\mathbf{S 3}$ presents all phantom experiment results obtained with

160 the vUS, CD-fUS, and PD-fUS analysis methods.

161 We further performed in vivo validation by comparing the velocity measured with ultrasound 162 localization microscopy velocimetry (vULM, Materials and Methods) against vUS, as shown in

163 Figure 3. We note that the measured axial velocity (Figure 3a1) and total velocity (Figure 3b1)

agree well between vUS and vULM. The weighted scatter plots of all nonzero pixels between vUS 
and vULM in Figure 3a2\&b2 indicate that the vUS measurement is highly correlated with the

vULM measurement. We further compared the mean velocity of 50 vessels marked in Figure S4

within the rodent brain.
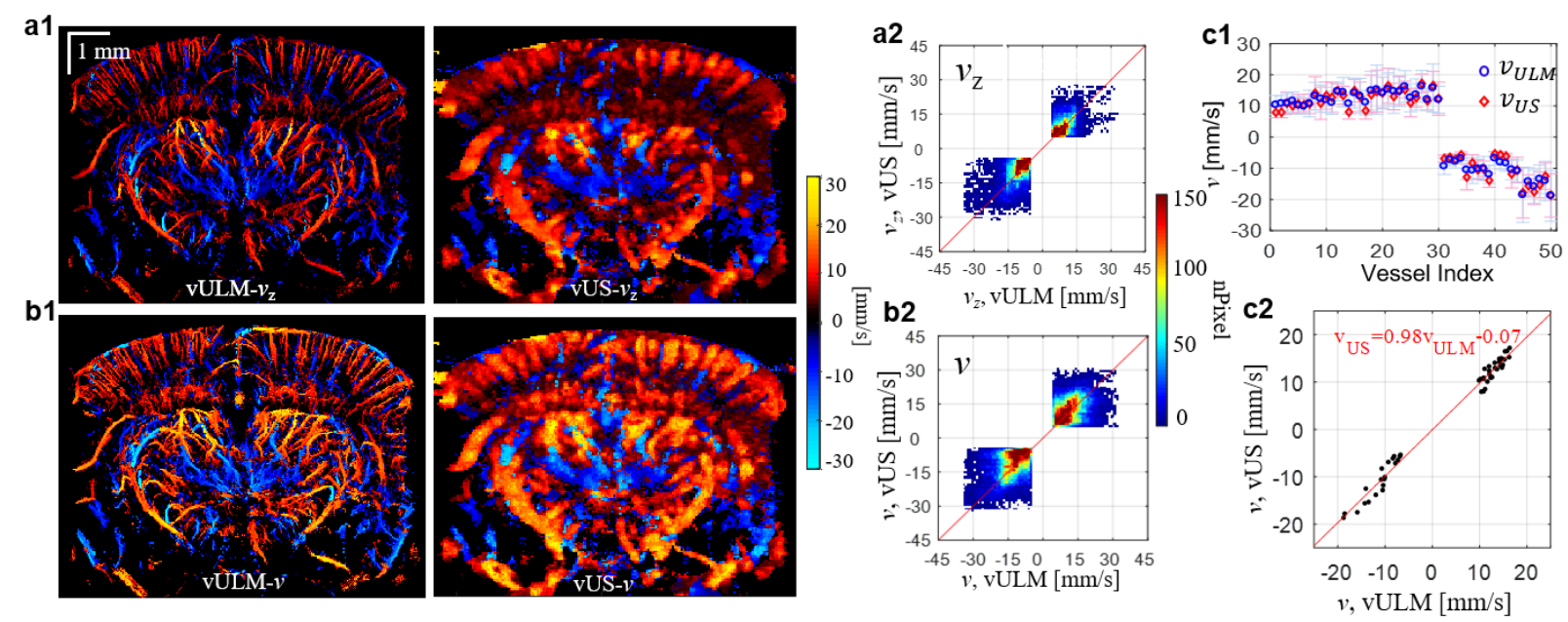

Figure 3 in vivo validation between vULM and vUS of axial velocity (a) and total velocity (b). (a2) and (b2) are pixel-to-pixel weighted scatter plot of common pixels of vULM and vUS with value $|v|>3 \mathrm{~mm} / \mathrm{s}$. (c1)

Mean velocity and standard deviation measured with vULM (blue) and vUS (red) of 50 vessels marked in and vUS $(\mathrm{r}=0.984, \mathrm{p}<0.001)$.

\subsection{Blood flow velocity change evoked by whisker stimulation}

To demonstrate the functional imaging capability of vUS, we measured the blood flow velocity response to whisker stimulation. We developed an animal preparation protocol using a 
183 in awake mice (Materials and Methods), as shown in Figure 4a\&b. Following the published
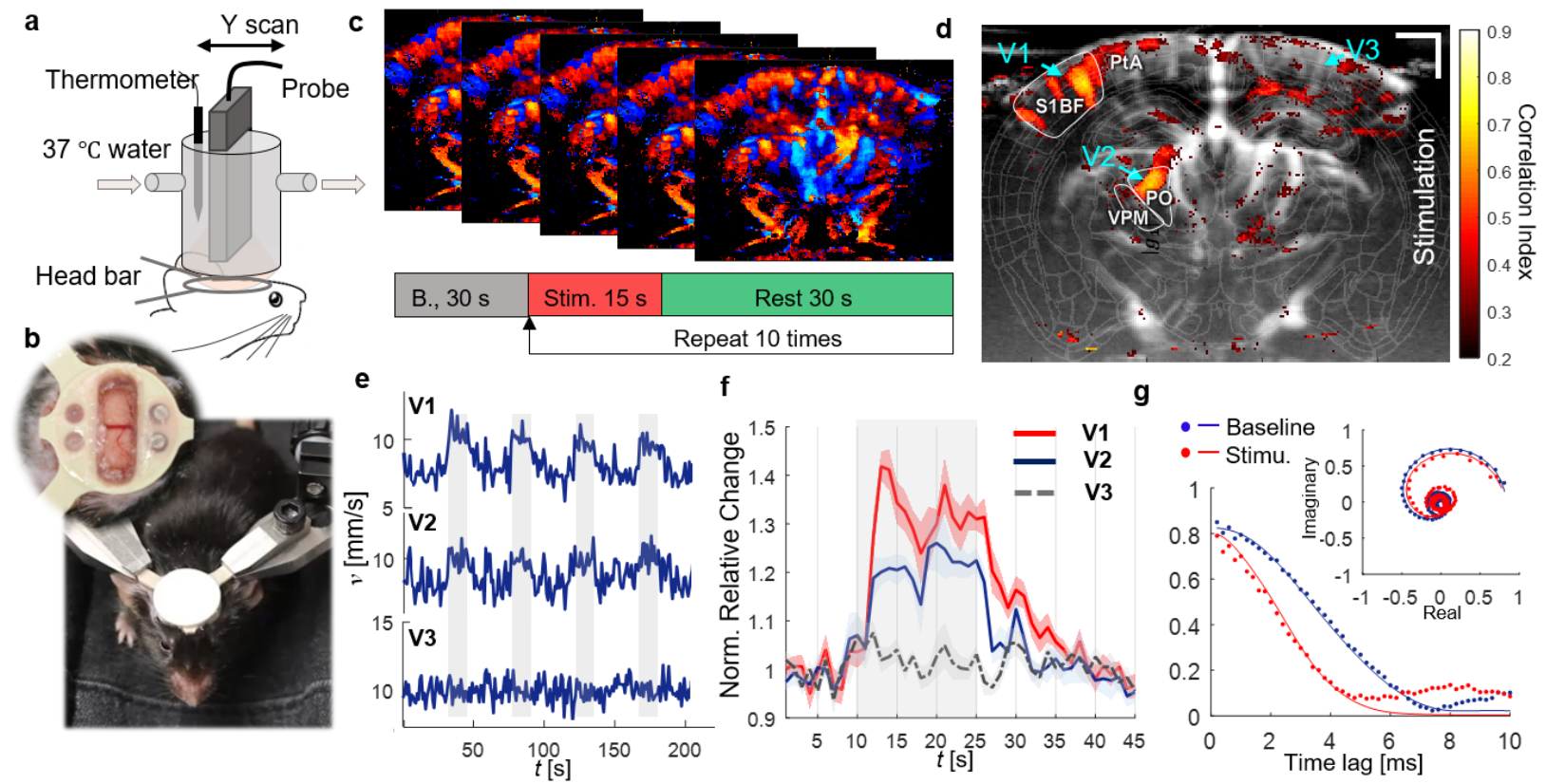

f

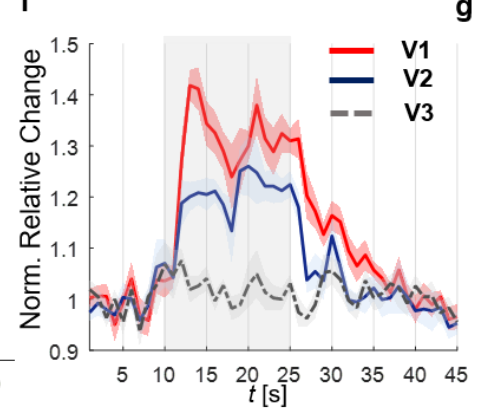

g

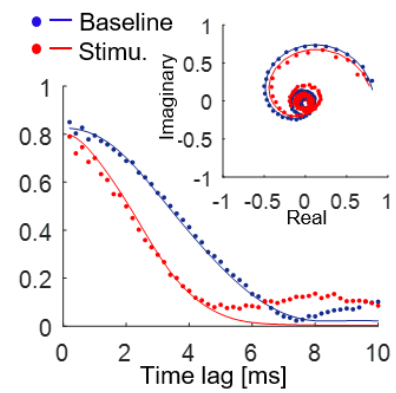

Figure 4 vUS of functional brain activation in awake mice. (a) Experimental setup. (b) Photos showing the trained mouse for awake-head fixed ultrasound imaging; inset: a PMP film protected cranial window was prepared in the center of the head bar for ultrasound imaging. (c) Whisker stimulation protocol and the vUS images were acquired at 1 frame/s. (d) Activation map in response to the mouse's left whisker stimulation. posteromedial nucleus of the thalamus; PtA: Posterior parietal association. The ROIs were identified according to Allen Mouse Brain Atlas(16). (e) First 4 trials of blood flow velocity time course of vessels V1, V2, and V3 as marked in (d). The voxels of the three vessel ROIs were selected with absolute velocity value greater than $3 \mathrm{~mm} / \mathrm{s}$. Gray shades indicate when stimulation was on. (f) Average blood flow velocity relative change of the 10 trials for the three vessels. Error bar: standard error of the mean. (g) Representative 
Figure 4d shows the correlation coefficient map between the blood flow velocity measured with

which shows no correlation with the stimulation. The Supplemental Video 1 shows the relative vUS is also sensitive to short duration stimulation evoked cerebral hemodynamic changes.

\subsection{Comparison of vUS with PD-fUS and CD-fUS}

220 The data set acquired for the vUS calculation can also be used for PD-fUS and CD-fUS data 
222 processing are apparent as shown in Figure 5. We see that 1) CD-fUS is only able to measure the

223 axial velocity component (Figure 5a); 2) the signal intensity of PD-fUS is not linearly related to

224 total speed but nonlinearly decreases with increasing speed (Figure 5a2\&b2); and 3) vUS is able

225 to measure the blood flow velocity of both angled (Figure 5a) and transverse (Figure 5b) flows

226 and differentiate the axial velocity component from the transverse velocity component (Figure

227 5a2), indicating the advantages of vUS in quantitatively imaging flow speeds in both axial and

228 transverse directions.

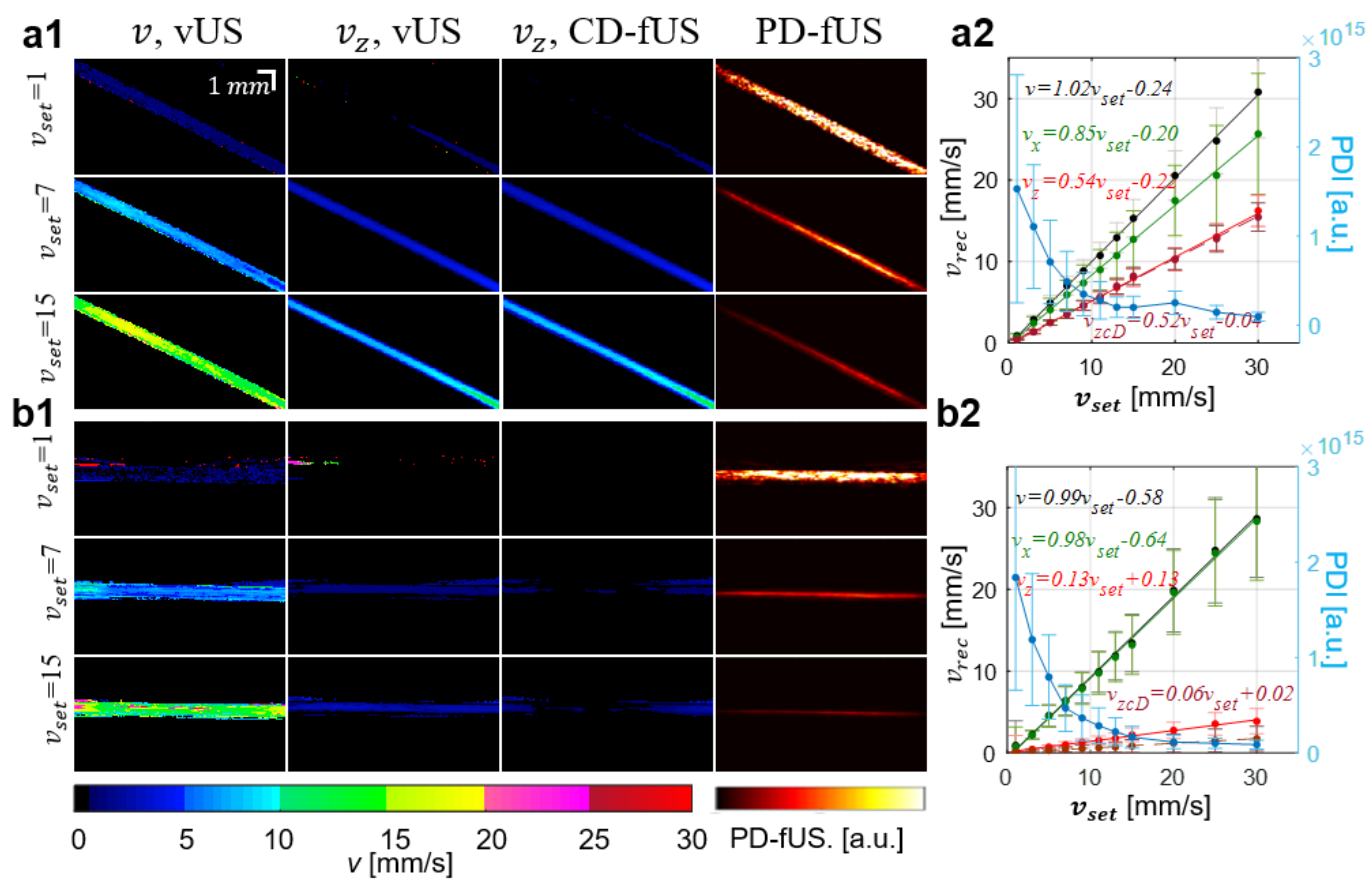

230 Figure 5 | Phantom results comparison of vUS with Power Doppler-based fUS (PD-fUS) and Color 231 Doppler-based fUS (CD-fUS). Angled (a) and transverse (B) flow phantom experiment results obtain with 232 vUS ( $v$ and $\left.v_{z}\right)$, CD-fUS $\left(v_{z}\right)$, and PD-fUS.

233 Figure 6a compares the in vivo measurements of ascending flow (positive frequency component) 234 obtained with vUS and PD-fUS. Using the vULM measurement as the comparison standard of flow 235 velocity, we note that vUS agrees well with vULM, while PD-fUS has high signal intensity in 
236 superficial layers and low signal intensity in deep regions, as indicated by the white and red arrows,

237 indicating the strong dependence of the PD-fUS signal on acoustic attenuation. In contrast, vUS is

238 not affected by acoustic attenuation as the normalization processing cancels the heterogeneous

239 acoustic distribution. Figure $6 \mathbf{b 1}$ shows the axial velocity maps obtained with conventional CD-

$240 \mathrm{fUS}^{[4]}$ (Online Methods). The conventional CD-fUS suffers from underestimation of Doppler

241 frequency $\left(f_{D}\right)$ due to mutual frequency cancellation when opposite flows exist within a

242 measurement voxel, as illustrated in Figure 6b2. For a fair comparison between vUS and the

243 Doppler methods, we applied CD-fUS processing on the directional filtered data that we used for

244 vUS processing. As shown in Figure 6c, we note that the blood flow speed is overestimated by the

245 directional filtering-based CD-fUS. This overestimation happens because of high frequency noise

246 causing overestimation of the Doppler frequency $\left(f_{D}\right)$ when a directional filter is applied and thus

247 a higher speed bias, as shown in Figure 6c2. In comparison, vUS doesn't suffer from the high

248 frequency noise as the high frequency noise is un-correlated and only causes $g_{1}(\tau)$ to drop to a

249 lower value at the first time lag but it doesn't affect the decorrelation rate of $g_{1}(\tau)$ at longer time

250 lags, which is determined by the correlated motion of flowing red blood cells, as shown in the

251 bottom panel of Figure $6 \mathbf{d} 2$. Thus, by fitting the decorrelation of $g_{1}(\tau)$ the blood flow velocity can

252 be accurately reconstructed by vUS, as shown in Figure 6d1. 

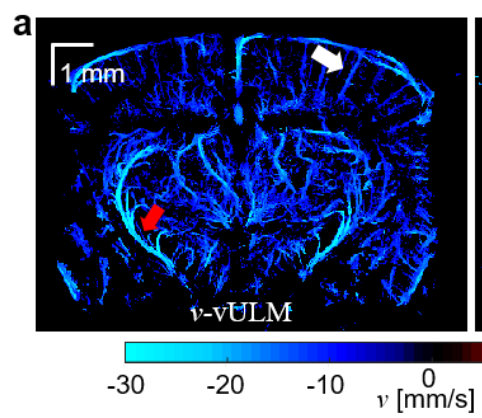

b1

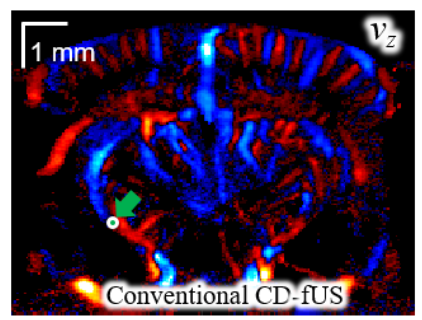

b2

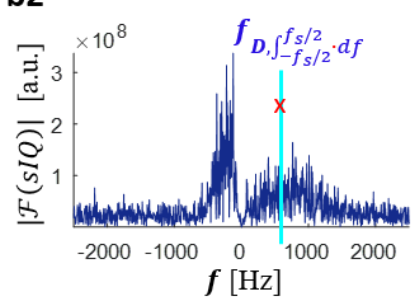

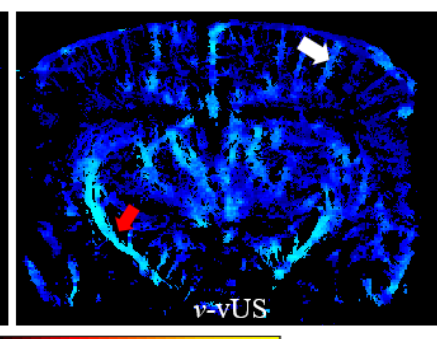

$10 \quad 20 \quad 30$

c1

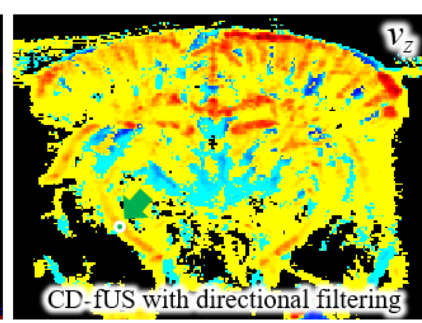

c2

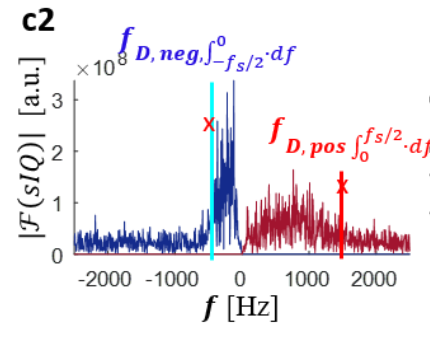

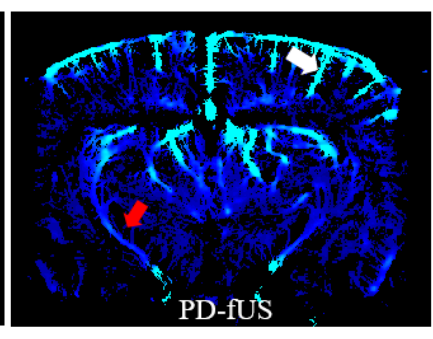

aScnd

PDI [a.u.]

d1

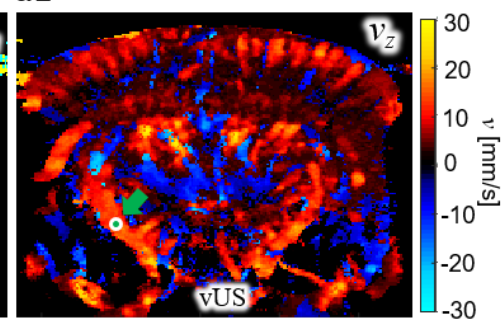

d2

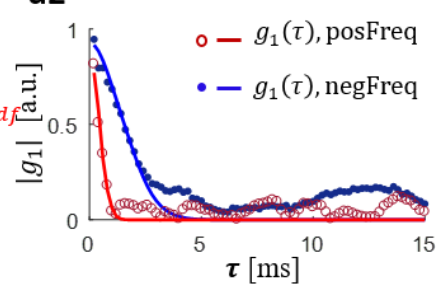

Figure 6 | in vivo results comparison. (a) in vivo ascending flow results obtained with vULM, vUS, and

PD-fUS, where vULM is used as the comparison standard and the ULM spatial mask was applied to both

$257\left(f_{D}\right)$ is underestimated with conventional CD-fUS. (c1) Axial velocity map obtained with directional

258 filtering-based CD-fUS; (c2) Doppler frequencies $\left(f_{D, n e g}\right.$ and $\left.f_{D, p o s}\right)$ are overestimated with the directional

259 filtering-based CD-fUS. (d1) Axial velocity map obtained with vUS; (d2) $g_{1}(\tau)$ calculated with positive

260 frequency component and negative frequency component after directional filtering; dots: experimental data;

261 solid line: theoretical fitting. Descending flow velocity maps were overlapped on ascending flow velocity

262 maps in (c1) and (d1).

\section{3. Discussion}


264 The development of robust blood flow velocity measurement technologies has been of great

265 importance in neuroscience research as quantifying blood flow alterations enables the assessment

266 of brain disease ${ }^{[17-19]}$ and interpretation of regional neural function according to neurovascular

267 coupling ${ }^{[20]}$. In this work, we introduced vUS based on the first-order temporal field autocorrelation

268 function analysis of the ultrasound speckle fluctuations to quantify cerebral blood flow velocity

269 with a temporal resolution of 1 frame/s (up to 5 frames/s in theory), with a greater than $10 \mathrm{~mm}$

270 penetration depth, and $\sim 100 \mu \mathrm{m}$ spatial resolution. vUS provides much deeper penetration

271 compared to optical velocimetry methods which are usually restricted to superficial layers of less

272 than $1 \mathrm{~mm}$ depth ${ }^{[21]}$ while maintaining high spatial and temporal resolution compared to magnetic

273 resonance imaging-based phase contrast velocity mapping ${ }^{[22]}$.

274 Using ultrasound signal decorrelation analysis to estimate flow speed dates back to the 1970s.

275 Atkinson and Berry ${ }^{[23]}$ have shown that the motion of moving scatterers is encoded in the

276 fluctuations of the ultrasound signal and Bamber et al. ${ }^{[24]}$ demonstrated that the ultrasound signal

277 decorrelation could be used to image tissue motion and blood flow. Wear and Popp and others ${ }^{[8,9,25-}$

$278{ }^{28]}$ showed that the decorrelation of ultrasound signal decays following a Gaussian form. In this

279 paper, we showed that the ultrasound signal field decorrelation is governed by three terms,

280 including the flow speed, the gradient of the axial velocity, and an axial velocity-dependent phase

281 term. This phase term gives vUS the ability to differentiate the axial velocity component from the

282 transverse velocity component.

283 The high frame rate ultrafast ultrasound plane-wave emission and acquisition paves the way for

284 vUS implementation, which permits the speckle decorrelation caused by the moving scattering

285 particles to be resolved with sufficiently high temporal resolution required to capture the speckle

286 decorrelation within the small measurement voxels. The combination of spatiotemporal singular 
value decomposition and high pass filtering plays an important role in rejecting bulk motion which enables the decorrelation of $g_{1}(\tau)$ to represent the dynamics of the motion of red blood cells and reconstructs both descending and ascending flow velocities from the negative frequency component and positive frequency component by applying directional filtering, respectively. We further developed a comprehensive fitting algorithm to reconstruct axial and transverse blood flow velocities. The proposed vUS technique was validated with numerical simulation, phantom experiments, and in vivo blood flow velocities obtained with vULM. The functional whisker stimulation experiment result agrees with previous rodent functional studies that mechanoreceptive whisker information reaches the barrel cortex via the thalamic VPM nuclei ${ }^{[29]}$, and the PO is a paralemniscal pathway for whisker signal processing ${ }^{[30]}$. This experiment demonstrates that vUS is sensitive to quantify the cerebral blood flow velocity change in response to functional stimulation and can be applied for brain imaging in awake mice.

flow velocity while the PD-fUS signal decreased with increasing speed and is strongly affected by the acoustic attenuation. Compared to CD-fUS (Color Doppler), vUS is able to measure both axial and transverse flow velocities and is resistant to high frequency noise compared to the directional

304 filtering-based CD-fUS which suffers from large or random values in regions with a low signal-tonoise ratio. Compared to vULM, vUS has lower spatial resolution but has much higher temporal resolution (up to $5 \mathrm{~Hz}$ of vUS compared to $2 \mathrm{mins} /$ frame of vULM) and is applicable for awake functional studies in rodents requiring high temporal resolution. In addition, it measures the flow velocity of the intrinsic contrast of red blood cells while vULM measures the speed of microbubbles. vUS is that the metabolic rate of oxygen can be quantitatively estimated if vUS measurements are 
311 combined with quantitative oxygenation measurements using multispectral photoacoustic

312 tomography ${ }^{[31,32]}$, providing a new high resolution biomarker for neuroscience research.

313 A limitation is that vUS is not sensitive to measuring blood flow velocity in small vessels with low

314 flow speeds due to the use of the spatiotemporal filter which rejects slow dynamics from the signal.

315 Also, limited by the spatial resolution of the ultrasound system, the reconstructed blood flow

316 velocity of a measurement voxel may represent integrated dynamics of multiple vessels that flow

317 through the measurement voxel. For the results presented in this work, vUS was simplified to

318 estimate in-plane 2D velocities (i.e., $v_{x}$ and $v_{z}$ ), ignoring decorrelation rom flow in the y-direction

319 (see Materials and Methods for justification). This simplification, however, results in a moderate

320 overestimation of the transverse velocity $\left(v_{x}\right)$ as $v_{x}$ tends to compensate for the decorrelation

321 caused by $v_{y}$. Nevertheless, we note that the measured total velocity is very close to that obtained

322 with vULM as shown in Figure 3. In the future, with the development of fast 3D ultrasound

323 imaging technology using a 2D transducer matrix, vUS can be easily adopted for 3D velocimetry

324 of the whole rodent brain.

\section{4. Experimental Section}

\section{1. vUS theory derivation}

327 The complex ultrasound quadrature signal of particles moving at the same speed in a measurement

328 voxel can be written as,

$$
\operatorname{sIQ}\left(x_{0}, y_{0}, z_{0}, t\right)=R \sum_{i_{S}}^{N_{S}} e^{-\frac{\left(x_{i_{S}}(t)-x_{0}\right)^{2}}{2 \sigma_{x}^{2}}-\frac{\left(y_{i_{S}}(t)-y_{0}\right)^{2}}{2 \sigma_{y}^{2}}-\frac{\left(z_{i_{S}}(t)-z_{0}\right)^{2}}{2 \sigma_{z}^{2}}} e^{i 2 k_{0}\left(z_{i_{S}}(t)-z_{0}\right)}
$$

330 Considering the basic scenario that all scatters have identical dynamics, i.e. the scatters are moving 


$$
\operatorname{sIQ}\left(x_{0}, y_{0}, z_{0}, t+\tau\right)=R \sum_{i_{S}}^{N_{S}} e^{-\frac{\left(x_{i_{S}}(t)+v_{x} \tau-x_{0}\right)^{2}}{2 \sigma_{x}^{2}}-\frac{\left(y_{i_{S}}(t)+v_{y} \tau-y_{0}\right)^{2}}{2 \sigma_{y}^{2}}-\frac{\left(z_{i_{S}}(t)+v_{z} \tau-z_{0}\right)^{2}}{2 \sigma_{Z}^{2}}} e^{i 2 k_{0}\left(z_{i_{S}}(t)+v_{z} \tau-z_{0}\right)}
$$

According to Equation 2, $g_{1}(\tau)$ for particles flowing identically within the ultrasound

measurement voxel can be derived to be,

$$
g_{1}(\tau)=e^{-\frac{v_{x} \tau^{2}}{4 \sigma_{x}^{2}}-\frac{v_{y} \tau^{2}}{4 \sigma_{y}^{2}}-\frac{v_{z} \tau^{2}}{4 \sigma_{z}^{2}}} e^{i 2 k_{0} v_{z} \tau}
$$

For microvasculature imaging of the rodent brain, the group velocity and velocity distribution must distributed flow,

$$
P\left(v_{x}, v_{y}, v_{z}\right)=\frac{1}{\pi \sqrt{\pi} \sigma_{v x} \sigma_{v y} \sigma_{v z}} e^{-\frac{\left(v_{x}-v_{x g p}\right)^{2}}{\sigma_{v x}^{2}}-\frac{\left(v_{y}-v_{y g p}\right)^{2}}{\sigma_{v y}^{2}}-\frac{\left(v_{z}-v_{z g p}\right)^{2}}{\sigma_{v z}^{2}}}
$$
describes the velocity distribution.

$g_{1}(\tau)=\sqrt{\frac{64 \sigma_{x}^{2} \sigma_{y}^{2} \sigma_{z}^{2}}{\left(4 \sigma_{x}^{2}+\sigma_{v x}^{2} \tau^{2}\right)\left(4 \sigma_{y}^{2}+\sigma_{v y}^{2} \tau^{2}\right)\left(4 \sigma_{z}^{2}+\sigma_{v z}^{2} \tau^{2}\right)}} e^{-\frac{\left(v_{x g p}\right)^{2}}{4 \sigma_{x}^{2}+\sigma_{v x}^{2} \tau^{2}}-\frac{\left(v_{y g p}\right)^{2}}{4 \sigma_{y}^{2}+\sigma_{v y}^{2} \tau^{2}}-\frac{\left(v_{z g p} \tau\right)^{2}+4 \sigma_{Z}^{2} \sigma_{v Z}^{2}\left(k_{0} \tau\right)^{2}}{4 \sigma_{Z}^{2}+\sigma_{v z}^{2} \tau^{2}}} i 2 k_{0} \tau \frac{4 \sigma_{z}^{2} v_{z g p}}{4 \sigma_{Z}^{2}+\sigma_{v z}^{2} \tau^{2}}$

$3484 \sigma_{\leftrightarrow}^{2} \geq 50 \times 10^{-4} \mathrm{~mm}^{2}$, where ' $\leftrightarrow$ ' represents the coordinate direction (i.e., $x, y$ or $z$ ). Thus, the 349 theoretical equation of $g_{1}(\tau)$ can be further simplified to be,

$$
g_{1}(\tau)=e^{-\frac{\left(v_{x g p} \tau\right)^{2}}{4 \sigma_{x}^{2}}-\frac{\left(v_{y g p}\right)^{2}}{4 \sigma_{y}^{2}}-\frac{\left(v_{z g p} \tau\right)^{2}}{4 \sigma_{z}^{2}}} e^{-\sigma_{v z}^{2}\left(k_{0} \tau\right)^{2}} e^{i 2 k_{0} \tau v_{z g p}}
$$


where, $\sigma_{x}, \sigma_{y}$, and $\sigma_{z}$ are the Gaussian profile width at the $1 / e$ value of the maximum intensity of

352 the point spread function (PSF) in $x, y$, and $z$ directions, respectively; $v_{g p}$ is the group velocity; and

$353 \sigma_{v z}$ describes the axial velocity distribution; and $k_{0}$ is the wave number of the central frequency of

354 the transducer.

355

\section{2. vUS implementation}

\subsubsection{Coherent plane wave compounding-based data acquisition}

The ultrasound signal was acquired with a commercial ultrafast ultrasound imaging system (Vantage 256, Verasonics Inc. Kirkland, WA, USA) and a linear ultrasonic probe (L22-14v, Verasonics Inc. Kirkland, WA, USA). The Vantage 256 system has 256 parallelized emission and receiving channels, and can acquire planar images at a frame rate up to $30 \mathrm{kHz}$ when the imaging depth is $\sim 15 \mathrm{~mm}$. The L22-14v ultrasonic probe has 128 transducer elements with a pitch of 0.1 $\mathrm{mm}$ and a center frequency of $18.5 \mathrm{MHz}$ with a bandwidth of $12.4 \mathrm{MHz}(67 \%,-6 \mathrm{~dB})$. It has an elevation focus at $\mathrm{z}=6 \mathrm{~mm}$.

To ensure sufficient temporal resolution, the ultrasound plane wave frame rate was set to $30 \mathrm{kHz}$ which was mainly limited by the transmit time of the ultrasound signal in the sample through the intended imaging depth, as shown in Figure S1a. To enhance the signal-to-noise ratio while preserving sufficient temporal resolution, we further employed coherence plane wave compounding ${ }^{[33]}$ at five emitting angles $\left(-6^{\circ},-3^{\circ}, 0^{\circ}, 3^{\circ}, 6^{\circ}\right)$ to form a compounded image whose frame rate was $5 \mathrm{kHz}$, as shown in Figure $\mathbf{S 1 b}$.

In addition, to acquire sufficient ensemble averaging of the US speckle fluctuations for the vUS analysis, we acquired $200 \mathrm{~ms}$ of data, i.e. 1,000 compounded images, to calculate $g_{1}(\tau)$ over a range of $0<\tau<20 \mathrm{~ms}$. Therefore, the maximum vUS frame rate is 5 frames/s. However, for extended 
data acquisition (i.e. $>1$ mins) the maximum vUS frame rate was reduced to $1 \mathrm{frame} / \mathrm{s}$ due to limit data transfer and saving requirements.

\subsubsection{Clutter rejection}

376 For the phantom data processing, we used a spatiotemporal filtering method (singular value

$$
\operatorname{sIQ}=\sum_{i=N c}^{N} S(z, x) \lambda_{i} V(t)
$$

where, sIQ is the dynamic signal; $N c$ is the cutoff rank for SVD processing; $S(z, x)$ is the spatial singular vector.

\subsection{3. vUS fitting algorithm}

387 Figure S1d summarizes the vUS data processing algorithm. Based on the developed vUS theory

$$
\mathcal{F}(s I Q)=\mathcal{F}_{\text {neg }}(s I Q)+\mathcal{F}_{\text {pos }}(s I Q)
$$

$$
s I Q_{\text {neg }}=\mathcal{F}^{-1}\left[\mathcal{F}_{\text {neg }}(s I Q)\right], \quad s I Q_{\text {pos }}=\mathcal{F}^{-1}\left[\mathcal{F}_{\text {pos }}(s I Q)\right]
$$


394 where, $s I Q_{n e g}$ and $s I Q_{p o s}$ are the complex ultrasound quadrature signal of the negative frequency

395 and positive frequency, respectively; $\mathcal{F}$ denotes the Fourier transform; and $\mathcal{F}^{-1}$ denotes the inverse

396 Fourier transform. $g_{1_{\text {neg }}}(\tau)$ and $g_{1_{\text {pos }}}(\tau)$ for $s I Q_{n e g}$ and $s I Q_{\text {pos }}$ are obtained using Equation 2,

397 respectively.

398 We used criteria including the ratio of positive/negative frequency power to whole frequency power

399 (Equation 13) and the absolute value of $g_{1}(\tau)$ at the first time lag (Equation 14) to control signal

400

quality for data processing.

401

$$
R_{\text {pos }}=\frac{\sum \mathcal{F}(\mathrm{sIQ})_{f>0}}{\sum \mathcal{F}(\mathrm{sIQ})_{\text {all freq. }}}>0.2, \quad R_{\text {neg }}=\frac{\sum \mathcal{F}(\mathrm{sIQ})_{f<0}}{\sum \mathcal{F}(\mathrm{sIQ})_{\text {all freq. }}}>0.25
$$

$$
\left|g_{1}(1)\right|>0.2
$$

where, $\mathcal{F}$ denotes the Fourier transform. These criteria enable us to skip the poor quality data,

which also greatly reduces the processing time.

Then, the fitting procedure is applied for both $s I Q_{n e g}$ and $s I Q_{p o s}$, respectively. In practice, random noise results in a prompt 'drop' of $g_{1}(1)$, i.e. the change of $g_{1}(0)$ to $g_{1}(1)$ is not a smooth transition compared to $g_{1}(1)$ to the end of the decorrelation as the noise is uncorrelated. We therefore modified the $g_{1}(\tau)$ equation by using an ' $\mathrm{F}$ ' factor to account for this 'drop'. Also, it is worth noting that when using a linear transducer array the ultrasound PSF is anisotropic in the transverse directions, i.e. $\sigma_{x} \neq \sigma_{y}$. In our experimental setup, $\sigma_{y}$ was more than 3 times larger than processing. In addition, in the case of Gaussian velocity distribution, $\sigma_{v z}$ is proportional to the 
415 Equation 3 can be replaced with $\sigma_{v z}=p \cdot v_{z g p}$ where $\mathrm{p}$ is a linear factor with a range of [0 1].

416 Thus the theoretical $g_{1}(\tau)$ model used for fitting the experimental data is,

$$
g_{1}(\tau)=F \cdot e^{-\frac{\left(v_{x g p} \tau\right)^{2}}{4 \sigma_{x}^{2}}-\frac{\left(v_{z g p} \tau\right)^{2}}{4 \sigma_{z}^{2}}} e^{-\left(p \cdot v_{z g p} \cdot k_{0} \cdot \tau\right)^{2}} e^{i 2 k_{0} \tau v_{z g p}}
$$

where F represents the correlated dynamic fraction which accounts for the $g_{1}(\tau)$ value drop at the of the transducer.

A proper initial guess of the unknown parameters (i.e., F, $v_{x g p}, v_{z g p}$, and $p$ ) is important to achieve

$$
v_{z g p 0}=\frac{\lambda_{0}}{4 \tau_{V}}
$$

We tested a mesh of $v_{x g p}$ and $p$ values to determine the initial guess of $v_{x g p 0}$ and $p_{0}$ by finding the

on the initial guesses.

$$
R=1-\frac{\langle| g_{1} \exp (\tau)-\left(F \cdot e^{-\frac{\left(v_{x g p} \tau\right)^{2}}{4 \sigma_{x}^{2}}-\frac{\left(v_{z g p} \tau\right)^{2}}{4 \sigma_{z}^{2}}} e^{-\left(p \cdot v_{z g p} \cdot k_{0} \cdot \tau\right)^{2}} e^{\left.\left.i 2 k_{0} \tau v_{z g p}\right)\left.\right|^{2}\right\rangle}\right.}{\left\langle\left|g_{1_{\exp }}(\tau)-\left\langle g_{1_{\exp }}(\tau)\right\rangle\right|\right\rangle^{2}}
$$


where, $g_{1_{\text {exp }}}(\tau)$ is the experimental $g_{1}(\tau)$ calculated with Equation $2 ;\langle\ldots\rangle$ indicates temporal ensemble averaging; and $|. .$.$| indicates the absolute value.$

438 Finally, the axial and total velocity maps were obtained for both descending and ascending flows, as shown in Figure S1e.

\subsection{Power Doppler-fUS and Color Doppler-fUS calculation}

The Power Doppler image (PD-fUS) was calculated as ${ }^{[4]}$,

$$
P D I=\frac{1}{N} \sum_{i=1}^{N} s I Q^{2}\left(t_{i}\right)
$$

where, $N$ is the number of samples and sIQ is the complex ultrasound quadrature signal of the moving particles.

The axial velocity based on the conventional Color Doppler calculation is obtained with ${ }^{[10]}$,

$$
v_{C Z}=-\frac{c}{2 f_{0}} \frac{\left.\int_{-f_{S} / 2}^{f_{S} / 2} \cdot|\mathcal{F}(s I Q)|^{2}\right) d f}{\left.\int_{-f_{S} / 2}^{f_{S} / 2}|\mathcal{F}(s I Q)|^{2}\right) d f}
$$

where, $c$ is the sound speed in the medium and $c=1540 \mathrm{~m} / \mathrm{s}$ was used in this study; $f_{0}$ is the transducer center frequency; $f_{s}$ is the frame rate; and $\mathcal{F}$ denotes the Fourier transform. obtain descending and ascending speeds (Figure 6c1),

$$
v_{c z, d s n d}=-\frac{c}{2 f_{0}} \frac{\left.\int_{-f_{S} / 2}^{0} f \cdot|\mathcal{F}(s I Q)|^{2}\right) d f}{\left.\int_{-f_{S} / 2}^{0}|\mathcal{F}(s I Q)|^{2}\right) d f}, \quad v_{c z, \text { asnd }}=-\frac{c}{2 f_{0}} \frac{\left.\int_{0}^{f_{S} / 2} f \cdot|\mathcal{F}(s I Q)|^{2}\right) d f}{\left.\int_{0}^{f_{S} / 2}|\mathcal{F}(s I Q)|^{2}\right) d f}
$$

\subsection{Ultrasound Localization Microscopy}


The images of the microbubble were rescaled to have a pixel size of $10 \mu \mathrm{m} \times 10 \mu \mathrm{m}$. The centroid position for each microbubble was then identified with $10 \mu \mathrm{m}$ precision by deconvolving the system point spread function. By accumulating the centroid positions over time, a high resolution image of the cerebral vasculature image (ULM) is obtained. Further, by identifying and tracking the same microbubble's position, the in-plane flow velocity of the microbubble can be calculated based on the travel distance and the imaging frame rate. The final velocity for coordinates $(z, x)$ consists of descending and ascending flows, and the speed for each direction was obtained by averaging the same directional flow speed at all time points when the absolute value was greater than 0 , respectively.

\subsection{Numerical Simulation}

467 In this study, two dimensional (x-z) flow and ultrasound detection was simulated to validate vUS.

468 Point scattering particles (5 $\mu \mathrm{m}$ in diameter) were randomly generated at the initialization segment which is outside the ultrasound measurement voxel. Then the flowing positions were calculated for all time points based on the preset flow speed and flow angle at a temporal rate of $5 \mathrm{KHz}$. The detected ultrasound signal $(s I Q)$ was obtained based on Equation 1 for each time point. Then the simulated $g_{1}(\tau)$ was calculated according to Equation 2 with 1000 observation time points (i.e. $200 \mathrm{~ms}$ ) and 100 autocorrelation calculation time lags (i.e. $20 \mathrm{~ms}$ ). Flow velocity was then reconstructed by applying vUS processing on the simulated $g_{1}(\tau)$.

\subsection{Phantom experiment and data processing}

476 For the phantom validation experiment, a plastic micro tube (inner diameter $580 \mu m$, Intramedic

477 Inc.) was buried in a homemade agar phantom with an angle of $\sim 30^{\circ}$ (angled flow), and another 478 plastic micro tube was aligned close to $\sim 0^{\circ}$ (transvers flow) in another homemade agar phantom. 479 A blood solution was pumped through the tubes with a syringe pump (Harvard Apparatus) at speeds 
of $1,3,5,7,9,11,13,15,20,25$, and $30 \mathrm{~mm} / \mathrm{s}$. SVD was performed to filter the background signal uniform. Therefore, the linear value $\mathrm{p}$ in Equation 15 was set to 0 (i.e. $\sigma_{v z}=0$ ) for the phantom data processing.

\subsection{Animal preparation}

486 The animal experiments were conducted following the Guide for the Care and Use of Laboratory

Animals, and the experiment protocol was approved by the Institutional Animal Care and Use

In this study, 12-16-week old C57BL/6 mice (22-28g, male, Charles River Laboratories) were used.

Animals were housed under diurnal lighting conditions with free access to food and water. Mice

PEEK headbar was attached to the skull using dental acrylic and bone screws. The skull between

\section{8. in vivo experiment and data processing}




\subsubsection{Experimental setup}

504 Agarose phantom (no scattering) was used to fill the cranial window, which serves as the acoustic matching medium between a water container and the mouse brain. The bottom of the water container was covered with a thin clear film preventing water leakage. To maintain the brain temperature of experimental animal, degassed warm water $\left(37^{\circ} \pm 1^{\circ}\right)$ was circulating through the water container and, along with the agarose phantom, worked as the acoustic transmitting medium between the ultrasound transducer and the mouse brain, as shown in Figure 4a. An anteroposterior linear translating stage was used to carry the ultrasound probe to acquire data at different coronal planes.

For anesthetized imaging, the experimental animal was anesthetized by isoflurane through a nose cone while the body temperature was maintained at $37^{\circ}$ with a homeothermic blanket control unit (Harvard Apparatus) and its head was fixed by a stereotaxic frame. For awake imaging, the experimental animal head was fixed by attaching the head-bar to a customized mount and the animal was treated with milk every $\sim 30 \mathrm{~min}$.

\subsubsection{In vivo validation}

For in vivo validation, animals were anesthetized with isoflurane and the body temperature was maintained at $37^{\circ}$. vUS data was first acquired at different coronal planes and followed by microbubble injection for ULM/vULM imaging for each coronal plane. $0.03 \mathrm{ml}$ commercial microbubble suspension (5.0-8.0 $\times 10^{8}$ microbubbles per ml, Optison, GE Healthcare, Milwaukee, rescaled to have the same pixel size $\left(25 \times 25 \mu m^{2}\right)$ as vUS map. For a fair comparison, both the 


\subsubsection{Whisker stimulation}

$527 \mathrm{~N}=3$ mice were trained and used for the whisker stimulation experiment. An air puffer machine 528 (Picospritzer III, Parker Inc.) was used for the whisker stimulation experiments. The outlet of the 529 air tube was placed $\sim 15 \mathrm{~mm}$ behind the whiskers. Two stimulation patterns were used in this study:

530 the first stimulation pattern (Figure 4 and Figure S5a) consisted of $30 \mathrm{~s}$ baseline and followed by 53110 trials of $15 \mathrm{~s}$ stimulation and with a $45 \mathrm{~s}$ interstimulus interval, and the second stimulation pattern 532 (Figure S5b) consisted of 20 s baseline and followed by 10 trials of 5 s stimulation and with a 25 533 s interstimulus interval. A motion correction method was used to replace the signal value at strong motion time points with the median value of adjacent time points. The stimulation frequency was $3 \mathrm{~Hz}$. the blood flow velocity $v(z, x, t)$ and the temporal stimulus pattern $S(t)$.

$$
r(z, x)=\frac{\sum_{t=1}^{N}(v(z, x, t)-\overline{v(z, x)})(S(t)-\bar{S})}{\sqrt{\sum_{t=1}^{N}(v(z, x, t)-\overline{v(z, x)})^{2}} \sqrt{\sum_{t=1}^{N}(S(t)-\bar{S})^{2}}}
$$

where,

$$
\overline{v(z, x)}=\frac{1}{N} \sum_{t=1}^{N} v(z, x, t) \text { and } \bar{S}=\frac{1}{N} \sum_{t=1}^{N} S(t)
$$

$$
z=\frac{\sqrt{N-3}}{2} \cdot \ln \frac{1+r}{1-r}
$$

\section{Supporting Information}

546 Supporting Information is available from the Wiley Online Library or from the author.

\section{Acknowledgements}


550

551

552

553

554

555

556

557

558

559

560

561

562

563

564

565

566

567

568

569

570

571

572

573

574

575

576

577

578

579

580

581

The authors acknowledge funding from NIH R01-EB021018, R01 NS108472, and R01MH111359.

\section{Competing financial interests}

The authors declare no competing financial interests.

\section{Authors contributions}

J.T. and D.A.B. conceived of the technology and designed this study. J.T., D.D.P., T.L.S. and D.A.B. developed the theoretical model and analyzed the results. J.T. derived the theoretical formula, developed the data processing method, constructed the experimental setup, carried out experiments, and wrote the manuscript. K.K., E.E. and B.L. developed the surgical protocol for chronic imaging on awake mice, carried out animal experiments and analyzed the results. J.T.G designed the head bar. D.A.B. supervised this study. All authors discussed the results and contributed to the final version of the manuscript.

\section{References}

[1] C. Poelma, Exp. Fluids 2017, 58, 3.

[2] J. A. Jensen, S. I. Nikolov, A. C. H. Yu, D. Garcia, IEEE Trans. Ultrason. Ferroelectr. Freq. Control 2016, 63, 1722.

[3] M. Tanter, M. Fink, IEEE Trans. Ultrason. Ferroelectr. Freq. Control 2014, 61, 102.

[4] E. MacÉ, G. Montaldo, I. Cohen, M. Baulac, M. Fink, M. Tanter, Nat. Methods 2011, 8, 662.

[5] B. F. Osmanski, S. Pezet, A. Ricobaraza, Z. Lenkei, M. Tanter, Nat. Commun. 2014, 5, DOI 10.1038/ncomms6023.

[6] L. A. Sieu, A. Bergel, E. Tiran, T. Deffieux, M. Pernot, J. L. Gennisson, M. Tanter, I. Cohen, Nat. Methods 2015, 12, 831.

[7] A. Urban, C. Dussaux, G. Martel, C. Brunner, E. Mace, G. Montaldo, Nat. Methods 2015, 12,873 . 
[8] L. Y. L. Mo, R. S. C. Cobbold, IEEE Trans. Biomed. Eng. 1992, 39, 450.

583

584

585

586

587

588

589

590

591

592

593

594

595

596

597

598

599

600

601

602

603

604

605

606

[9] R. S. Adler, J. M. Rubin, J. B. Fowlkes, P. L. Carson, J. E. Pallister, Ultrasound Med. Biol. 1995, 21, 493.

[10] T. L. Szabo, Diagnostic Ultrasound Imaging : Inside Out, Elsevier Science, Oxford, n.d.

[11] J. M. Rubin, R. O. Bude, P. L. Carson, R. L. Bree, R. S. Adler, Radiology 1994, 190, 853.

[12] J. A. Jensen, S. I. Nikolov, A. C. H. Yu, D. Garcia, IEEE Trans. Ultrason. Ferroelectr. Freq. Control 2016, 63, 1704.

[13] C. Errico, J. Pierre, S. Pezet, Y. Desailly, Z. Lenkei, O. Couture, M. Tanter, Nature 2015, $527,499$.

[14] P. Song, J. D. Trzasko, A. Manduca, R. Huang, R. Kadirvel, D. F. Kallmes, S. Chen, IEEE Trans. Ultrason. Ferroelectr. Freq. Control 2017, 3010, 1.

[15] N. Uribe-Patarroyo, B. E. Bouma, Phys. Rev. E 2016, 94, 1.

[16] C. Ayata, A. K. Dunn, Y. Gursoy-Özdemir, Z. Huang, D. A. Boas, M. A. Moskowitz, J. Cereb. Blood Flow Metab. 2004, 24, 744.

[17] B. V. Zlokovic, Nat. Rev. Neurosci. 2011, 12, 723.

[18] K. Kisler, A. R. Nelson, A. Montagne, B. V. Zlokovic, Nat. Rev. Neurosci. 2017, 18, 419.

[19] C. Iadecola, Neuron 2013, 80, 844.

[20] C. Iadecola, Nat. Rev. Neurosci. 2004, 5, 347.

[21] A. Devor, S. Sakadžic, V. J. Srinivasan, M. A. Yaseen, K. Nizar, P. A. Saisan, P. Tian, A. M. Dale, S. A. Vinogradov, M. A. Franceschini, D. A. Boas, J. Cereb. Blood Flow Metab. 2012, 32, 1259.

[22] P. Chai, R. Mohiaddin, 2005, DOI 10.1081/JCMR-200065639.

[23] P. Arkinson, M. V Berry, Related Content Diffractal Echoes, 1974.

[24] J. C. Bamber, P. Hasan, G. Cook-Martin, N. Bush, J Ultrasound Med 1988, 7.

[25] K. A. Wear, R. L. Popp, IEEE Trans. Med. Imaging 1987, 6, 281. 
607

608

609

610

611

612

613

614

615

616

617

618

619

620

621

622

623

624

625

[26] J. M. Rubin, T. A. Tuthill, J. B. Fowlkes, Ultrasound Med. Biol. 2001, 27, 101.

[27] X. Zhou, C. H. Leow, E. Rowland, K. Riemer, J. M. Rubin, P. D. Weinberg, M.-X. Tang, IEEE Trans. Ultrason. Ferroelectr. Freq. Control 2018, 65, 2233.

[28] J. Meunier, M. Bertrand, Ultrasonic Texture Motion Analysis: Theory and Simulation, 1995.

[29] D. Casas-Torremocha, F. Clascá, Á. Núñez, Front. Neural Circuits 2017, 11, 69.

[30] T. Pierret, P. Lavallée, M. Deschênes, J. Neurosci. 2000, 20, 7455.

[31] S. Tzoumas, A. Nunes, I. Olefir, S. Stangl, P. Symvoulidis, S. Glasl, C. Bayer, G. Multhoff, V. Ntziachristos, Nat. Commun. 2016, 7, DOI 10.1038/ncomms12121.

[32] G. Diot, S. Metz, A. Noske, E. Liapis, B. Schroeder, S. V. Ovsepian, R. Meier, E. Rummeny, V. Ntziachristos, Clin. Cancer Res. 2017, 23, 6912.

[33] G. Montaldo, M. Tanter, J. Bercoff, N. Benech, M. Fink, IEEE Trans. Ultrason. Ferroelectr. Freq. Control 2009, 56, 489.

[34] C. Demené, T. Deffieux, M. Pernot, B. F. Osmanski, V. Biran, J. L. Gennisson, L. A. Sieu, A. Bergel, S. Franqui, J. M. Correas, I. Cohen, O. Baud, M. Tanter, IEEE Trans. Med. Imaging 2015, 34, 2271. 


\section{Supporting Information}

\section{Functional ultrasound speckle decorrelation-based velocimetry of the brain}

\section{Supplementary Figures}
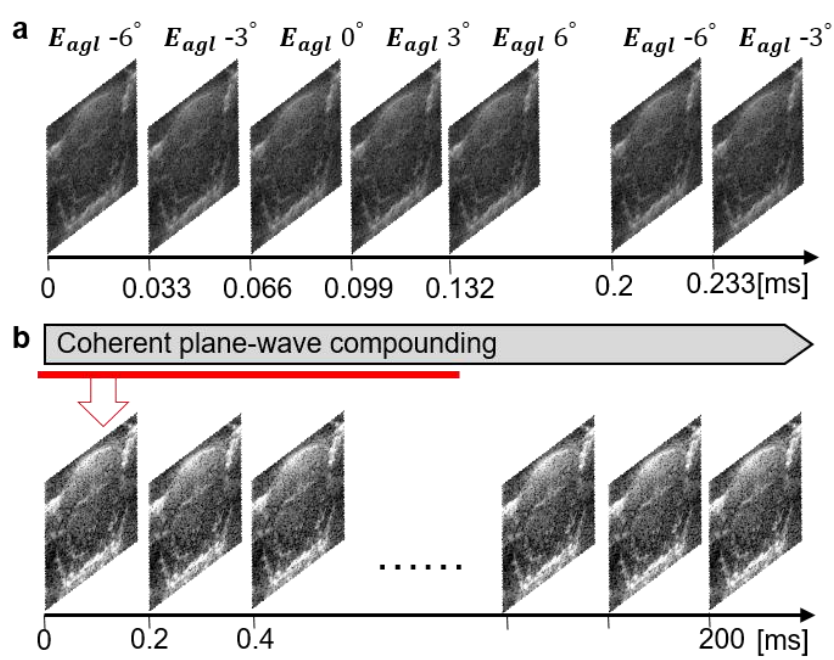

c Singular Value Decomposition \& High pass filtering

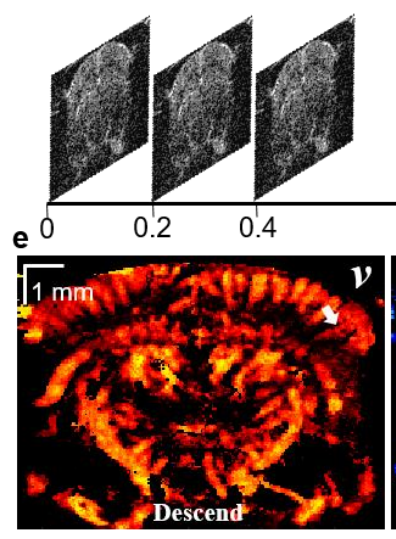

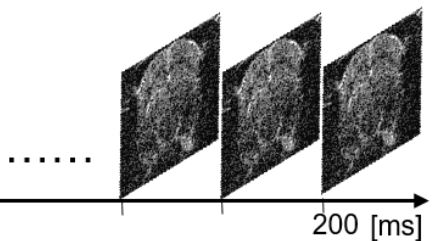

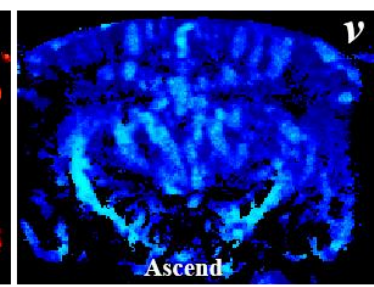

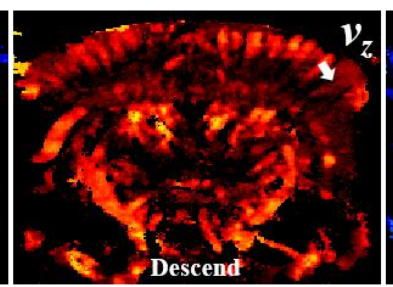
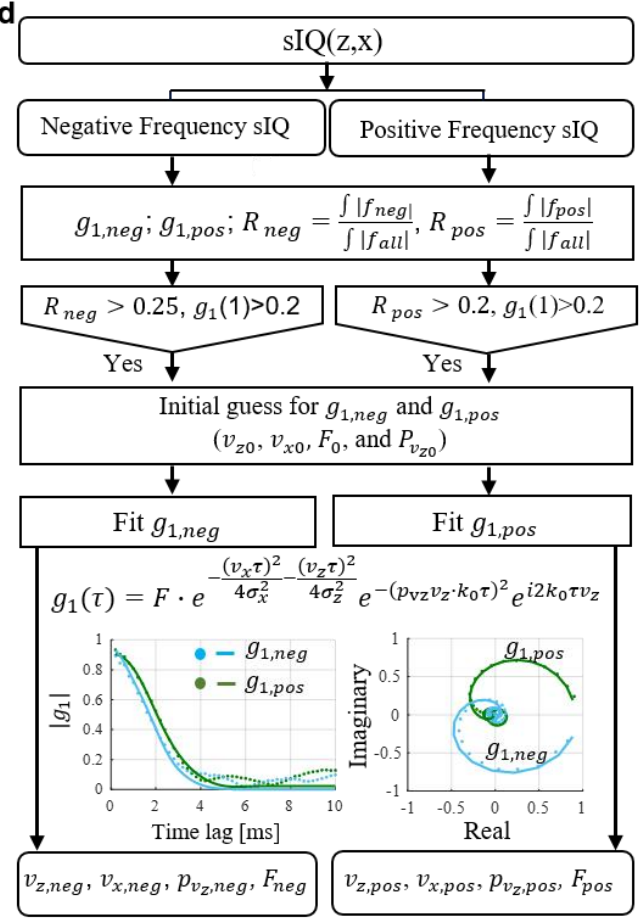

Figure S1 | vUS implementation and data processing. (a) Ultrasound pulse \& acquisition sequence. (b) Coherent plane-wave compounding were performed on the 5 tilted emission angle frames and produced a compounded image at a frame rate of $5 \mathrm{kHz}$. (c) Clutter rejection were performed to remove static background and bulk motion signal components. (d) Negative and positive frequency components of a measurement voxel are processed separately for in vivo data vUS processing; dots: experimental data; solid lines: fitting results. (e) Descending and ascending blood flow velocity maps reconstructed by vUS of a coronal plane ( Bregma $-2.18 \mathrm{~mm})$ of a mouse brain. 

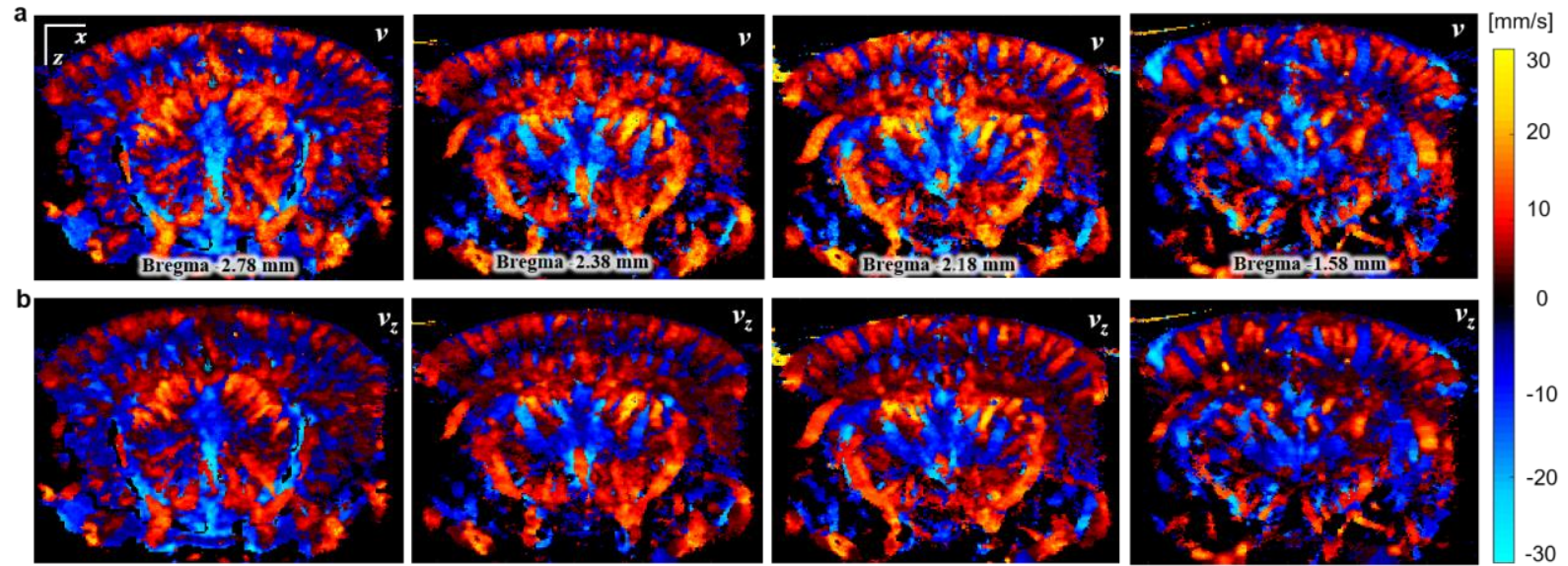

644 Figure S2 | Total velocity (a) and axial velocity (b) obtained with vUS at different coronal planes of a mouse
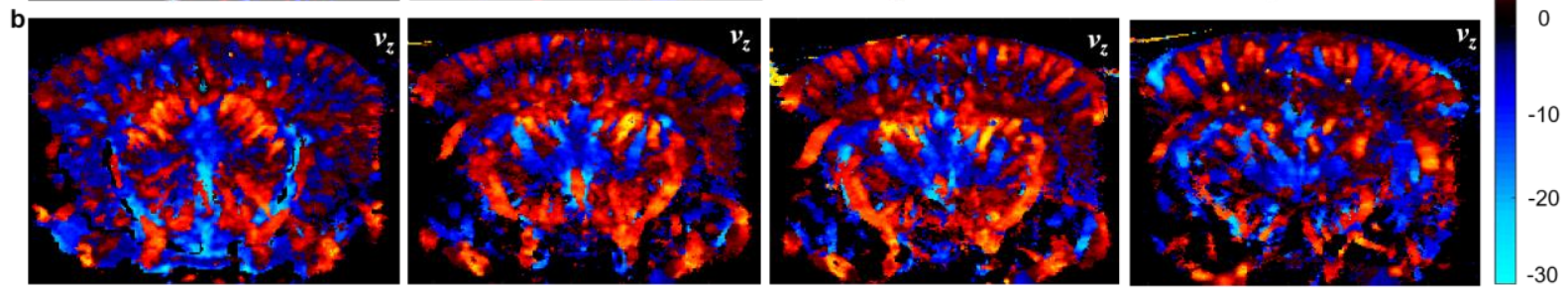

brain. Descending flow velocity map was overlapped on ascending flow velocity map.

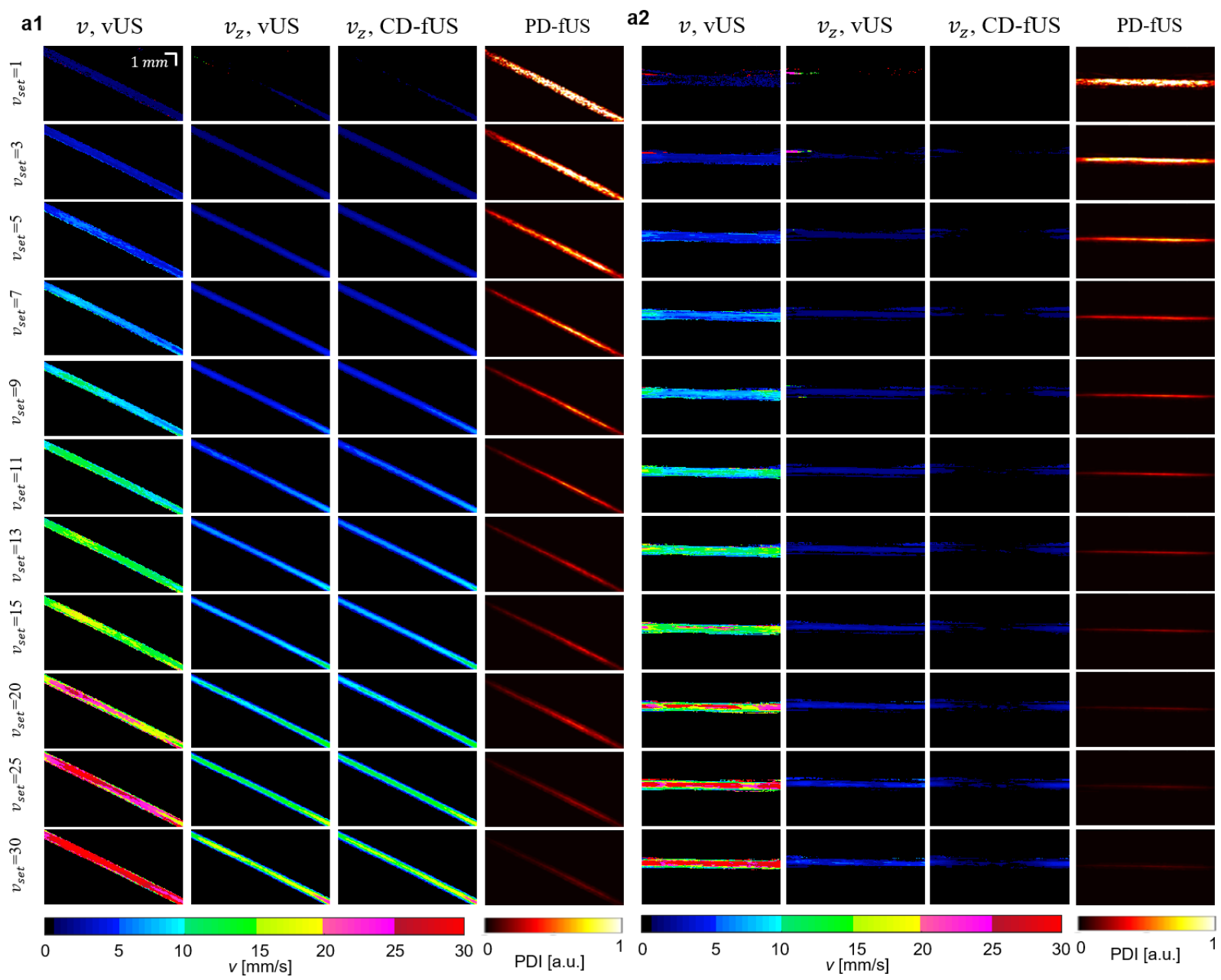

Figure S3 | Phantom experiment validation and comparison. (a) Results for angled flow phantom 648 experiments. (b) Results for transverse flow phantom experiments. vUS is able to accurately measure both 649 axial and transverse velocity components while CD-fUS is not capable of measuring the transverse flow 650 velocity component. In addition, vUS is able to accurately differentiate the axial velocity component from 

measured velocity has a linear relationship with the preset speeds, while the PD-fUS measured signal decreases nonlinearly with increasing preset speed.
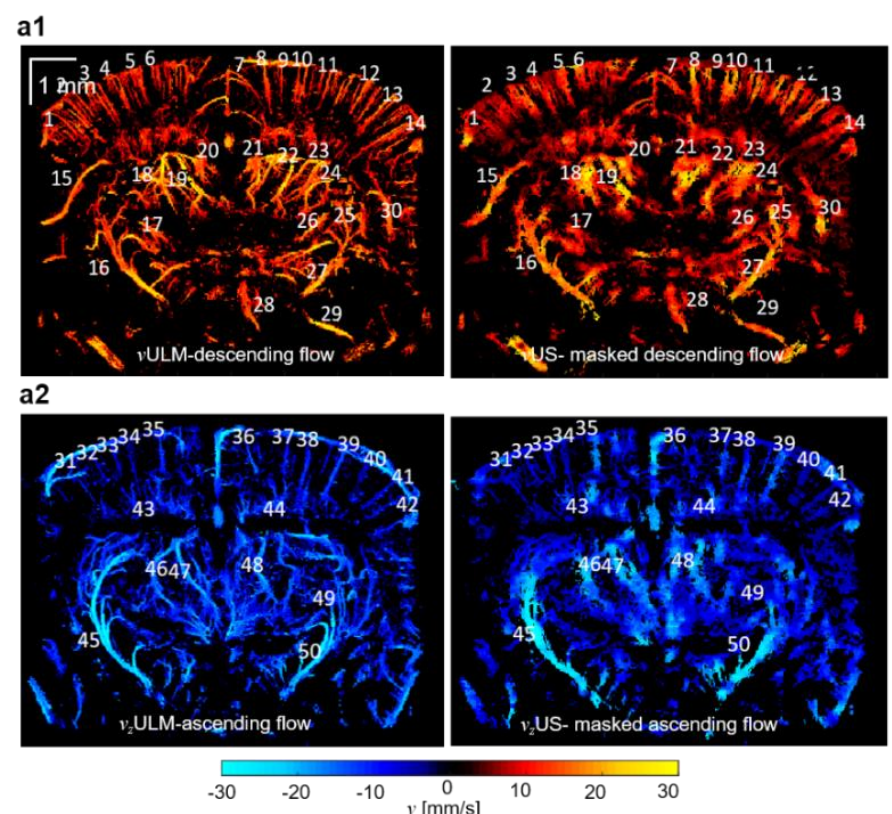
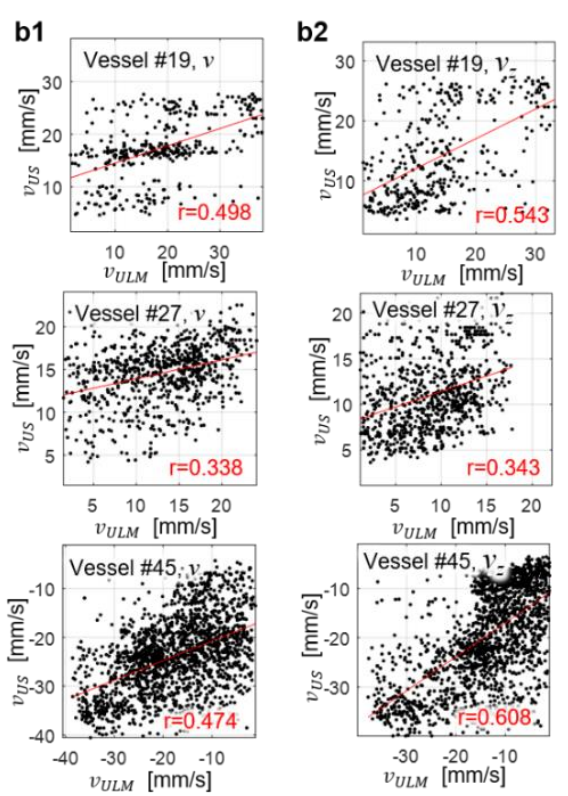

654

655

656

657

658

Figure S4 | in vivo validation by comparing vUS with vULM. (a) The numbers show the indices of selected vessel for vessel-to-vessel comparison between vUS and vULM. (b1) Scatter plots of total velocity of three representative vessels show the pixel-to-pixel correlation between vULM and vUS. (b2) Scatter plots of axial velocity of three representative vessels show the pixel-to-pixel correlation between vULM and vUS.

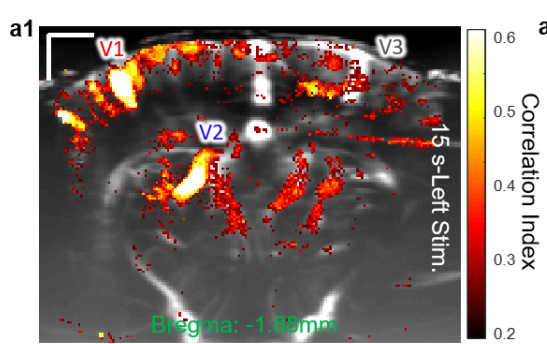

b1

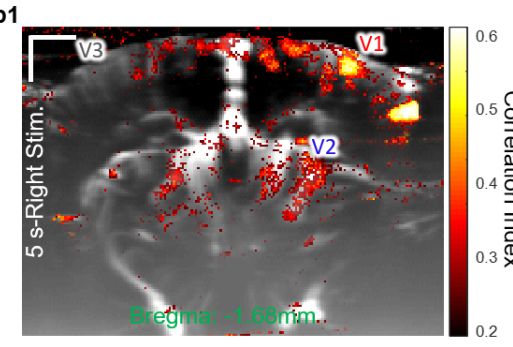

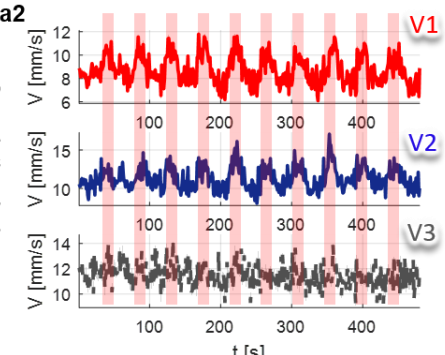

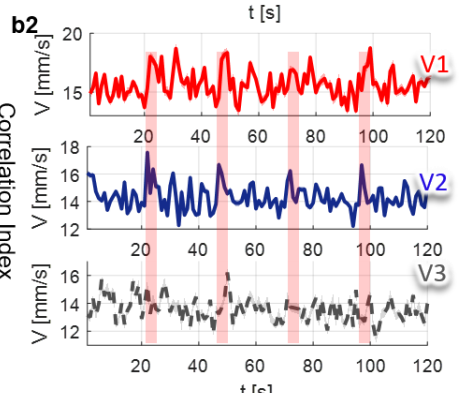

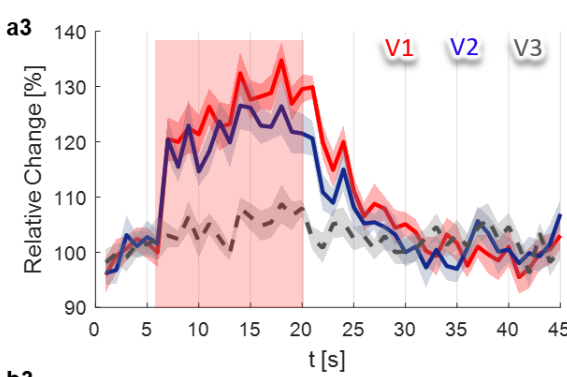

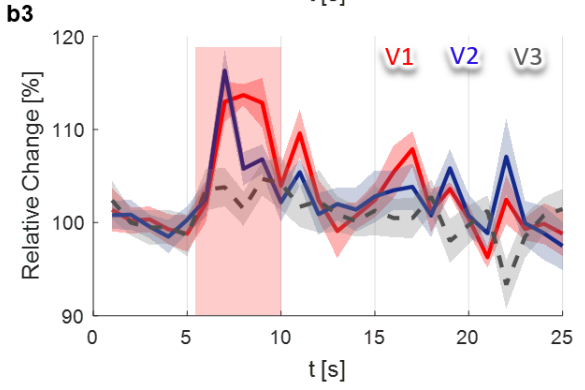

Figure S5 | Representative whisker stimulation results. (a) Results of 15 seconds left side whisker stimulation; (a1) Activation map; (a2) Blood flow velocity time courses for the three vessels marked in (a1); (a3) 10 trials averaged relative response of the three vessels. (b) Results of 5 seconds right side whisker stimulation at Bregma -1.58 mm; (b1) Activation map; (b2) Blood flow velocity time courses for the three vessels marked in (b1); (b3) 10 trials averaged relative response of the three vessels. 


\section{Function description for vUS data processing}

\section{Note: vUS data processing code and example data is available from: Supplementary \\ 667 Code.}

\section{A. vUS data processing for in vivo data}

\section{A.1. main function}

$\% \%$ IQ to vUS data processing for in vivo experiment

clear all; clc

addpath('./SubFunctions/');

$\% \%$ Use GPU calculation or not

useGPU = questdlg('Use GPU for data processing?', 'Select', ...

'YES', 'NO', 'Cancel', 'Cancel');

$\% \%$ Load data

disp(['Loading data, ', datestr(datetime('now'))]);

load ('./DATA/invivoData.mat');

[nz,nx,nt]=size(IQ);

PRSSinfo.g1StartT $=1$

PRSSinfo.g1nT=nt;

PRSSinfo.g1nTau $=100$;

PRSSinfo.rFrame $=5000 ; \%$ sIQ frame rate, $\mathrm{Hz}$

PRSSinfo.SVDrank=[25, nt $]$;

PRSSinfo.HPfC $=25 ; \%$ high pass frequency cutoff

PRSSinfo.FWHM=[125 100]*1e-6; \% (X, Z) spatial resolution, Full Width at Half Maximum of point spread function, $m$

PRSSinfo.C $=1540$; $\%$ sound speed, $\mathrm{m} / \mathrm{s}$

PRSSinfo.f0=16.625*1e6; \% Transducer center frequency, $\mathrm{Hz}$

PRSSinfo.xCoor=interp(P.xCoor,PRSSinfo.rfnScale);

PRSSinfo.zCoor=interp(P.zCoor,PRSSinfo.rfnScale);

PRSSinfo.MpVz=1; \% maximum p value for $\mathrm{SigmaVz}$

PRSSinfo.NEQ $=0 ; \%$ no noise equalization

$\% \% 1$. Clutter rejection

disp(['Clutter Rejection - ', datestr(datetime('now'))]);

[sIQ, sIQHP, sIQHHP, eqNoise]=IQ2sIQ(IQ(:,:,1:PRSSinfo.g1nT),PRSSinfo); \% 0: no noise equalization

$[\mathrm{nz}, \mathrm{nx}, \mathrm{nt}]=\operatorname{size}(\mathrm{sIQ})$;

clear IQ

$\% \% 2$. vUS data processing

disp(['g1-based vUS Processing - ', datestr(datetime('now'))]);

if strcmp(useGPU, 'YES')

disp('GPU-based vUS Processing ...(NOTE: it taks around 30 seconds)');

tic;[F, Vz, V, pVz, R]=sIQ2vUS_NPDV_GPU(sIQ, PRSSinfo);toc

else

disp('CPU-based vUS Processing ...(NOTE: it taks around 400 seconds)');

tic;[F, Vz, V, pVz, R]=sIQ2vUS_NPDV(sIQ, PRSSinfo);toc

end

$\% \% 3$. save results and plot $\mathrm{V}$ and $\mathrm{Vz}$

[VzCmap]=Colormaps_fUS;

save(['./vUS.mat'],'-v7.3','F','Vz','V','R','pVz');

$\operatorname{disp}([$ 'Results are saved! - ', datestr(datetime('now'))]);

$\%$ figure plot

Coor. $\mathrm{x}=$ PRSSinfo. $\mathrm{xCoor} ; \quad$ Coor. $\mathrm{z}=$ PRSSinfo.. $\mathrm{Coor}$;

Fig=figure;

set(Fig, 'Position',[300 4001300 400]);

subplot $(1,2,1)$

Fuse2Images(V(:,:,1),V(:,:,2),[-30 30],[-30 30],Coor.x,Coor.z,2.5);

title(['vUS, V [mm/s]']);

subplot $(1,2,2)$

Fuse2Images(Vz(:,:,1),Vz(:,:,2),[-30 30],[-30 30],Coor.x,Coor.z,2.5);

title(['vUS, Vz [mm/s]']);

\section{A.2. function IQ2sIQ}

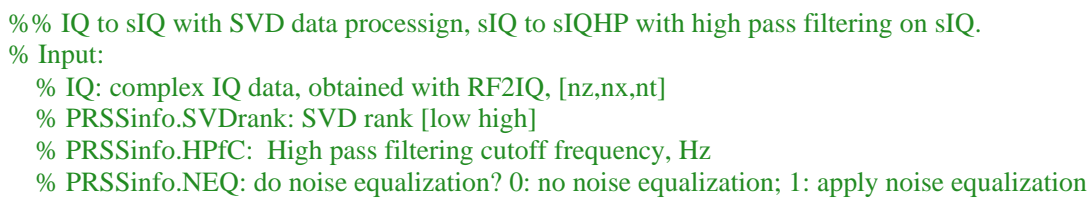


bioRxiv preprint doi: https://doi org/101101/686774; this version posted March 31, 2020. The copyright holder for this preprint (which was not certified by peer review) is the author/funder, who has granted bioRxiv a license to display the preprint in perpetuity. It is made available under aCC-BY 4.0 International license.

$\%$ PRSSinfo.rFrame: imaging frame rate, $\mathrm{Hz}$

$\%$ output:

$\%$ sIQ: SVD clutter rejected data, [nz,nx,nt]

$\%$ sIQHP: SVD+HP clutter rejected data, [nz,nx,nt], cutoff frequency: PRSSinfo.HPfC

$\%$ sIQHHP: SVD+HHP clutter rejected data, [nz,nx,nt], cutoff frequency: $70 \mathrm{~Hz}$

732

(unction:

$\%$ [sIQ, Noise]=SVDfilter(IQ,SignalRank)

function [sIQ, sIQHP, sIQHHP, eqNoise]=IQ2sIQ(IQ,PRSSinfo)

\section{A.3. function sIQ2vUS_NP_DV}

$\% \%$ US g1 fit for in vivo data, fit negative and postive frequency signal separately

$\%$ input:

$\%$ sIQ: bulk motion removed data, [nz,nx,nt]

$\%$ PRSSinfo: data processing parameters, including

\% PRSSinfo.FWHM: $(\mathrm{X}, \mathrm{Y}, \mathrm{Z})$ spatial resolution, Full Width at Half Maximum of point spread function, $\mathrm{m}$

$\%$ PRSSinfo.rFrame: sIQ frame rate, $\mathrm{Hz}$

$\%$ PRSSinfo.f0: Transducer center frequency, $\mathrm{Hz}$

$\%$ PRSSinfo.C: Sound speed in the sample, $\mathrm{m} / \mathrm{s}$

$\%$ PRSSinfo.g1nT: g1 calculation sample number

\% PRSSinfo.g1nTau: maximum number of time lag

$\%$ PRSSinfo.SVDrank: SVD rank [low high]

$\%$ PRSSinfo.HPfC: High pass filtering cutoff frequency, $\mathrm{Hz}$

$\%$ PRSSinfo.NEQ: do noise equalization? 0: no noise equalization; 1 : apply noise equalization

$\%$ PRSSinfo.rfnScale: spatial refind scale

$\%$ PRSSinfo.MpVz: maximu $\mathrm{pVz}$

\% PRSSinfo.useMsk: 1: use ULM data as spatial mask; 0: no spatial mask

$\%$ PRSSinfo.ulmMsk: ULM-based spatial constrain mask $\%$ output:

$\%$ F: dynamic component fraction, [nz,nx,2], 2: [real,imag]

$\%$ Vz: axial-direction velocity component, [nz,nx], mm/s

$\% \mathrm{~V}=\operatorname{sqrt}\left(\mathrm{Vx} .{ }^{\wedge} 2+\mathrm{Vz} .^{\wedge} 2\right),[\mathrm{nz}, \mathrm{nx}], \mathrm{mm} / \mathrm{s}$

$\% \mathrm{pVz}$ : Vz distribution (sigma-Vz), [nz,nx]

$\%$ R: fitting accuracy, [nz,nx]

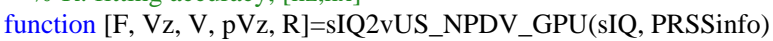

760

761

function $[\mathrm{F}, \mathrm{Vz}, \mathrm{V}, \mathrm{pVz}, \mathrm{R}]=\mathrm{sIQ} 2 \mathrm{vUS} \_N P D V(\mathrm{sIQ}$, PRSSinfo) 
bioRxiv preprint doi: https://doi.org/10.1101/686774; this version posted March 31, 2020. The copyright holder for this preprint (which was not certified by peer review) is the author/funder, who has granted bioRxiv a license to display the preprint in perpetuity. It is made available under aCC-BY 4.0 International license.

\section{B. vUS data processing (SV model) for phantom data}

763

\section{B.1. Main function}

$\% \%$ IQ to vUS data processing for ex vivo data using the basic model

clear all; clc

addpath('./SubFunctions');

$\% \%$ Use GPU calculation or not

useGPU = questdlg('Use GPU for data processing?', 'Select', ...

'YES', 'NO', 'Cancel', 'Cancel');

$\% \%$ Load data

$\operatorname{disp}([$ 'Loading data, ', datestr(datetime('now'))]);

$\%$ load ('./DATA/phantomData5a.mat'); \% angled flow, preset speed $5 \mathrm{~mm} / \mathrm{s}$

load ('./DATA/phantomData15a.mat'); \% angled flow, preset speed $15 \mathrm{~mm} / \mathrm{s}$

$\%$ load ('./DATA/phantomData9t.mat'); \% transverse flow, preset speed $9 \mathrm{~mm} / \mathrm{s}$

$\%$ load ('./DATA/phantomData25t.mat'); \% transverse flow, preset speed $25 \mathrm{~mm} / \mathrm{s}$

$\%$ IQ: beamformed complex quadratue data

$[\mathrm{nz}, \mathrm{nx}, \mathrm{nt}]=\operatorname{size}(\mathrm{IQ})$;

PRSSinfo.g1StartT=1;

PRSSinfo.g1nT=nt;

PRSSinfo.g1nTau $=100$;

PRSSinfo.rFrame=5000; \% sIQ frame rate, $\mathrm{Hz}$

PRSSinfo.SVDrank $=[3, \mathrm{nt}]$;

PRSSinfo.HPfC $=25$; $\%$ high pass frequency cutoff

PRSSinfo.FWHM=[125 100]*1e-6; \% (X, Z) spatial resolution, Full Width at Half Maximum of point spread function, m

PRSSinfo. $\mathrm{C}=1540$; $\%$ sound speed, $\mathrm{m} / \mathrm{s}$

$\%$ Transducer center frequency, $\mathrm{Hz}$

PRSSinfo.rfnScale=1;

PRSSinfo. $x$ Coor=interp(P.xCoor,PRSSinfo.rfnScale);

PRSSinfo.zCoor=interp(P.zCoor,PRSSinfo.rfnScale);

$\mathrm{PRSSinfo} . \mathrm{NEQ}=0$; $\%$ no noise equalization

$\% \%$ Clutter rejection

disp(['Clutter Rejection - ', datestr(datetime('now'))]);

[sIQ, sIQHP, sIQHHP, eqNoise]=IQ2sIQ(IQ(:,:,1:PRSSinfo.g1nT),PRSSinfo); \% 0: no noise equalization $[\mathrm{nz}, \mathrm{nx}, \mathrm{nt}]=\operatorname{size}(\mathrm{sIQ})$;

clear IQ

disp(['Power Doppler Processing - ', datestr(datetime('now'))]);

$[\mathrm{PDI}]=\mathrm{sIQ} 2 \mathrm{PDI}(\mathrm{sIQ}) ; \%$ PDI processing

disp(['Color Doppler Processing - ', datestr(datetime('now'))]);

$\mathrm{V} c z 0=($ ColorDoppler(sIQ,PRSSinfo $)) ; \%$ color Doppler, all frequency

$\operatorname{disp}([$ 'g1-based vUS Processing - ', datestr(datetime('now'))]);

if strcmp(useGPU, 'YES')

Dev=gpuDevice;

disp('GPU-based vUS Processing ...(NOTE: it taks around 4 seconds)');

tic;[F, Vz, Vx, V, R]=sIQ2vUS_SV_GPU(sIQ, PRSSinfo);toc

else

disp('CPU-based vUS Processing ...(NOTE: it taks around 30 seconds)'); end

$\mathrm{V} c z=$ imresize $(\mathrm{Vcz} 0,[\mathrm{nz}, \mathrm{nx}] *$ PRSSinfo.rfnScale,'bilinear').*CR;

save(['./vUS.mat'],'-v7.3','F','Vz','Vx','V','Vcz','R','PRSSinfo','P');

disp(['Results are saved! - ', datestr(datetime('now'))]);

$\% \%$ figure plot

[VzCmap,VzCmapDn, VzCmapUp, PhtmCmap]=Colormaps_fUS;

Coor. $\mathrm{x}=[1: \mathrm{nx}]^{*} 0.05 /$ PRSSinfo.rfnScale;

Coor. $z=[1: \mathrm{nz}]^{*} 0.05 /$ PRSSinfo.rfnScale;

Fig=figure;

set(Fig,'Position',[400 4001700 350])

subplot $(1,3,1)$

h1=imagesc(Coor.x,Coor.z,abs(V));

colormap(PhtmCmap);

caxis([0 30]);

colorbar

axis equal tight;

xlabel('x [mm]')

ylabel('z [mm]')

title('vUS-V $[\mathrm{mm} / \mathrm{s}]$ ')

subplot $(1,3,2)$

h2=imagesc(Coor.x,Coor.z,abs(Vz));

colormap(PhtmCmap); 
bioRxiv preprint doi: https://doi org/101101/686774; this version posted March 31, 2020. The copyright holder for this preprint (which was not

$\operatorname{caxis}([030])$

colorbar

axis equal tight;

xlabel('x $[\mathrm{mm}] ')$

ylabel('z [mm]')

title('vUS-Vz [mm/s]')

subplot $(1,3,3)$

h3=imagesc(Coor.x,Coor.z,abs(Vcz));

colormap(PhtmCmap);

$\operatorname{caxis}\left(\left[\begin{array}{ll}0 & 30]\end{array}\right)\right.$;

colorbar

axis equal tight;

xlabel('x [mm]')

ylabel('z [mm]')

title('Color Doppler-Vz [mm/s]')

847

848

\section{B.2. function sIQ2vUS_SV}

849

850

851

852

853

854

855

856

857

858

859

860

861

862

863

864

865

866

867

868

869

870

871

872

873

874

875

$\% \%$ US g1 fit, fit all frequency signal, for single flow direction scenario

$\%$ input:

$\%$ sIQ: bulk motion removed data

$\%$ PRSSinfo: data acquistion information, including

\% PRSSinfo.FWHM: (X, Y, Z) spatial resolution, Full Width at Half Maximum of point spread function, $\mathrm{m}$

$\%$ PRSSinfo.rFrame: sIQ frame rate, $\mathrm{Hz}$

\% PRSSinfo.fO: Transducer center frequency, $\mathrm{Hz}$

$\%$ PRSSinfo.C: Sound speed in the sample, $\mathrm{m} / \mathrm{s}$

\% PRSSinfo.g1nT: g1 calculation sample number

$\%$ PRSSinfo.g1nTau: maximum number of time lag

$\%$ PRSSinfo.SVDrank: SVD rank [low high]

$\%$ PRSSinfo.HPfC: High pass filtering cutoff frequency, $\mathrm{Hz}$

\% PRSSinfo.NEQ: do noise equalization? 0: no noise equalization; 1: apply noise equalization

$\%$ PRSSinfo.rfnScale: spatial refind scale

output:

$\%$ PRSSinfo.MpVz=0; \%

$\%$ F: dynamic factor

$\%$ Vz: axial velocity component, $\mathrm{mm} / \mathrm{s}$

$\% \mathrm{Vx}$, transverse velocity component, $\mathrm{mm} / \mathrm{s}$

$\%$ V: total velocity, $\mathrm{mm} / \mathrm{s}$

$\%$ R: fitting accuracy

$\%$ CR: vUS data processing criteria mask

function [F, Vz, Vx, V, R, CR]=sIQ2vUS_SV_GPU(sIQ, PRSSinfo);toc

function $[\mathrm{F}, \mathrm{Vz}, \mathrm{Vx}, \mathrm{V}, \mathrm{R}, \mathrm{CR}]=\mathrm{sIQ} 2 \mathrm{vUS} \_\mathrm{SV}(\mathrm{sIQ}, \mathrm{PRSSinfo})$;toc

\section{B.3. function ColorDoppler}

877

$\% \%$ color Doppler data processing to get axial blood flow velocity

$\%$ input:

$\%$ sIQ: bulk motion removed data

$\%$ PRSSinfo: data acquistion information, including

$\%$ PRSSinfo.rFrame: sIQ frame rate, $\mathrm{Hz}$

\% PRSSinfo.f0: Transducer center frequency, $\mathrm{Hz}$

$\%$ PRSSinfo.C: Sound speed in the sample, $\mathrm{m} / \mathrm{s}$

$\%$ output:

$\%$ Vcz: axial velocity calculated with Color Dopler, $\mathrm{mm} / \mathrm{s}$

885

886

function $[\mathrm{Vcz}]=$ ColorDoppler(sIQ,PRSSinfo) 